\title{
ON LAGRANGIAN SUBMANIFOLDS IN COMPLEX HYPERQUADRICS AND ISOPARAMETRIC HYPERSURFACES IN SPHERES
}

\author{
HUI MA AND YOSHIHIRO OHNITA \\ Dedicated to Professor Hajime Urakawa on his sixtieth birthday
}

\begin{abstract}
The $n$-dimensional complex hyperquadric is a compact complex algebraic hypersurface defined by the quadratic equation in the $(n+1)$ dimensional complex projective space, which is isometric to the real Grassmann manifold of oriented 2- planes and is a compact Hermitian symmetric space of rank 2. In this paper we study geometry of compact Lagrangian submanifolds in complex hyperquadrics from the viewpoint of the theory of isoparametric hypersurfaces in spheres. From this viewpoint we provide a classification theorem of compact homogeneous Lagrangian submanifolds in complex hyperquadrics by using the moment map technique. Moreover we determine the Hamiltonian stability of compact minimal Lagrangian submanifolds embedded in complex hyperquadrics which are obtained as Gauss images of isoparametric hypersurfaces in spheres with $g(=1,2,3)$ distinct principal curvatures.
\end{abstract}

\section{INTRODUCTION}

Let $(M, \omega)$ be a $2 n$-dimensional symplectic manifold with symplectic form $\omega$. The Lagrangian submanifold $L$ in $M$ is by definition an $n$-dimensional smooth submanifold $L$ in $M$ such that the pull-back of $\omega$ to $L$ vanishes. It is an interesting problem to investigate Lagrangian submanifolds in specific symplectic manifolds such as Kähler manifolds, complex space forms, Hermitian symmetric spaces, generalized flag manifolds with invariant symplectic forms, toric Kähler manifolds etc., from both viewpoints of symplectic geometry and Riemannian geometry (cf. Section 11).

In this paper we study compact Lagrangian submanifolds in complex hyperquadrics $Q_{n}(\mathbf{C})$. The $n$-dimensional complex hyperquadric $Q_{n}(\mathbf{C})$ is a compact complex algebraic hypersurface defined by the quadratic equation $z_{0}^{2}+z_{1}^{2}+\cdots+z_{n}^{2}+z_{n+1}^{2}=0$ in the $(n+1)$-dimensional complex projective space, which is isometric to the real Grassmann manifold of oriented 2dimensional vector subspaces of $\mathbf{R}^{n+2}$ and is a compact Hermitian symmetric space of rank 2. In Section 2, we discuss a link of Lagrangian Geometry in the

Date: August 10, 2008.

The first named author is partially supported by NSFC grant No. 10501028, SRF for ROCS, SEM and NKBRPC No. 2006CB805905. The second named author is partially supported by JSPS grant of Kiban (A) No. 17204006.

2000 Mathematics Subject Classification. Primary: 53C42; Secondary: 53C40, 53D12.

Key words and phrases. Lagrangian submanifold, minimal submanifold, Hamiltonian stability, symplectic geometry, Gauss map, isoparametric hypersurface. 
complex hyperquadrics with Hypersurface Geometry in the sphere. We know a fundamental fact that the Gauss map of any oriented hypersurface in the standard sphere $S^{n+1}(1)$ is always a Lagrangian immersion into the complex hyperquadric $Q_{n}(\mathbf{C})$. It follows from the mean curvature formula of B. Palmer ([37]) that the images of the Gauss map of compact oriented hypersurfaces with constant principal curvatures, so called isoparametric hypersurfaces, in spheres provide a nice class of compact minimal Lagrangian submanifolds embedded in complex hyperquadrics. Particularly homogeneous isoparametric hypersurfaces in spheres correspond to minimal Lagrangian orbits in complex hyperquadrics. The theory of isoparametric hypersurfaces was originated by Élie Cartan. We know that by the result of W. Y. Hsiang-J. B. Lawson ([16]) homogeneous isoparametric hypersurface can be obtained as a principal orbit of the isotropy representation of a compact Riemannian symmetric pair $(U, K)$ of rank 2. Since non-homogeneous isoparametric hypersurfaces in spheres exist (H. Ozeki-M. Takeuchi ([34, 35])) and are almost classified (D. FerusH. Karcher-H. F. Münzner ([12]), T. Cecil-Q. S. Chi-G. R. Jensen ([9])) at present, they also give many nice examples of compact non-homogeneous minimal Lagrangian submanifolds in complex hyperquadrics.

As the theory of isoparametric hypersurfaces are now well-developed in differential geometry, we are interested in the following problem :

Problem. Investigate properties of compact minimal Lagrangian submanifolds in complex hyperquadrics obtained as Gauss images of isoparametric hypersurfaces in spheres. More generally, study compact Lagrangian submanifolds in complex hyperquadrics by using the theory of isoparametric hypersurfaces in spheres.

A Lagrangian submanifold obtained as a Lagrangian orbit of the Hamiltonian group action on $M$ is called a homogeneous Lagrangian submanifold. The investigation of the Lie-theoretic construction of Lagrangian submanifolds is inspired by [32, 5]. In Section 3 we shall discuss Lie algebraic properties of homogeneous isoparametric hypersurfaces in spheres related with homogeneous Lagrangian geometry in complex hyperquadrics such as the moment maps.

In Section 4, based on the link with theory of homogeneous isoparametric hypersurfaces, we shall show a classification theorem of compact homogeneous Lagrangian submanifolds, i.e., Lagrangian orbits of compact connected Lie subgroups of $S O(n+2)$, in $Q_{n}(\mathbf{C})$. The moment map technique plays an essential role in the argument. We shall prove that all compact homogeneous Lagrangian submanifolds in $Q_{n}(\mathbf{C})$ are the Gauss image of compact homogeneous isoparametric hypersurfaces in spheres, or are obtained as their Lagrangian deformations in the following cases : Suppose that $(U, K)$ is one of

(a) $\left(S^{1} \times S O(3), S O(2)\right)$,

(b) $(S O(3) \times S O(3), S O(2) \times S O(2))$,

(c) $(S O(3) \times S O(n+1), S O(2) \times S O(n))(n \geq 3)$,

(d) $(S O(m+2), S O(2) \times S O(m))(n=2 m-2, m \geq 3)$.

In the first two cases, it is elementary and well-known to describe all Lagrangian orbits of the natural actions of $K=S O(2)$ on $Q_{1}(\mathbf{C}) \cong S^{2}$ and 
$K=S O(2) \times S O(2)$ on $Q_{2}(\mathbf{C}) \cong S^{2} \times S^{2}$. Also in the last two cases there exist one-parameter families of Lagrangian $K$-orbits in $Q_{n}(\mathbf{C})$ and each family contains Lagrangian submanifolds which can NOT be obtained as the Gauss image of any homogeneous isoparametric hypersurface in a sphere. The fourth one is a new family of Lagrangian orbits and it will be discussed in detail.

The notion of Hamiltonian minimality and Hamiltonian stability for Lagrangian submanifolds in Kähler manifolds was introduced and investigated first by Y. G. Oh ([29], [30], 31], [32]). A compact Lagrangian submanifold $L$ in a Kähler manifold $M$ is called Hamiltonian minimal (shortly, H-minimal) if it has extremal volume under every Hamiltonian deformations of $L$. A compact H-minimal Lagrangian submanifold in a Kähler manifold $M$ is called Hamiltonian stable (shortly, H-stable) if the second variation for the volume is nonnegative under every Hamiltonian deformations of $L$. We do NOT know so many examples of compact Hamiltonian stable Lagrangian submanifolds yet.

Problem. Construct and classify compact Hamiltonian stable minimal or Hminimal Lagrangian submanifolds in specific Kähler manifolds.

In Section 5 we shall determine Hamiltonian stability of compact minimal Lagrangian submanifolds embedded in $Q_{n}(\mathbf{C})$ obtained as Gauss images of isoparametric hypersurfaces with $g=1,2,3$. Due to the result of E. Cartan, they all are homogeneous. The Gauss images of compact isoparametric hypersurfaces with $g=2$ are not Hamiltonian stable if and only if the multiplicities $m_{1}, m_{2}$ of the principal curvatures satisfy $m_{2}-m_{1} \geq 3$. We shall show that the Gauss images of all compact isoparametric hypersurfaces with $g=3$ are Hamiltonian stable and they provide new examples of compact Hamiltonian stable minimal Lagrangian submanifolds embedded in $Q_{n}(\mathbf{C})$.

Acknowledgments. This work has been done during the second named author's visits at Tsinghua University in Beijing and Fudan University in Shanghai in 2005-2006 and the first named author's stay at Osaka City University Advanced Mathematical Institute (OCAMI) in 2007. The authors would like to thank Professors Zizhou Tang and Yuanlong Xin for helpful conversations and generous supports, and Professor Akio Kawauchi (Director of OCAMI) for excellent support and research circumstance based on OCAMI. They also would like to thank Professors Franz Pedit and Ulrich Pinkall for suggestion on the elementary version of Theorem 1.1.

\section{Hamiltonian Deformations of Lagrangian SUbmanifolds}

Let $(M, \omega)$ be a $2 n$-dimensional symplectic manifold with a symplectic form $\omega$. A Lagrangian immersion $\varphi: L \longrightarrow M$ is a smooth immersion of an $n$ dimensional smooth manifold $L$ into $M$ satisfying the condition $\varphi^{*} \omega=0$. Generally a smooth immersion $\varphi: Q \longrightarrow M$ of a $p$-dimensional smooth manifold $Q$ into $M$ satisfying the condition $\varphi^{*} \omega=0$ is called isotropic.

The normal bundle $\varphi^{-1} T M / \varphi_{*} T L$ of a Lagrangian immersion $\varphi: L \longrightarrow M$ can be identified with the cotangent bundle $T^{*} L$ of $L$ :

$$
\varphi^{-1} T M / \varphi_{*} T L \ni v \longmapsto \alpha_{v}:=\omega(v, \cdot) \in T^{*} L
$$


By the definition a Lagrangian deformation is a smooth family of Lagrangian immersions $\varphi_{t}: L \longrightarrow M$ with $\varphi=\varphi_{0}$. Let $\alpha_{V_{t}}$ be a 1-form on $L$ corresponding to its variational vector field

$$
V_{t}:=\frac{\partial \varphi_{t}}{\partial t} \in C^{\infty}\left(\varphi_{t}^{-1} T M\right)
$$

The Lagrangian deformation is characterized by the condition that $\alpha_{V_{t}}$ is closed, i.e., $\alpha_{V_{t}} \in Z^{1}(L)$, for each $t$. Furthermore, if $\alpha_{V_{t}}$ is exact, i.e., $\alpha_{V_{t}} \in$ $B^{1}(L)$, for each $t$, then $\left\{\varphi_{t}\right\}$ is called a Hamiltonian deformation of $\varphi=\varphi_{0}$.

Suppose that $[(1 / 2 \pi) \omega] \in H^{2}(M ; \mathbf{R})$ is an integral cohomology class. Then there is a complex line bundle $\mathcal{L}$ over $M$ with a $U(1)$-connection $\nabla$ whose curvature coincides with $\sqrt{-1} \omega$, and thus the Lagrangian property is equivalent to the flatness of the induced connection in the pull-back $U(1)$-bundle $\varphi^{-1} \mathcal{L}$. Then we can show

Theorem 1.1. A Lagrangian deformation $\left\{\varphi_{t}\right\}$ is a Hamiltonian deformation if and only if $\left\{\varphi_{t}\right\}$ provides an isomonodromy deformation of the induced flat $U(1)$-connections in $\varphi_{t}^{-1} \mathcal{L}$, that is, they have the same holonomy homomorphism $\pi_{1}(L) \rightarrow U(1)$.

Proof. For each $t$ let $\rho_{t}: \pi_{1}(L) \rightarrow U(1)$ denote the holonomy homomorphism of the induced flat $U(1)$-connection $\nabla^{t}=\varphi_{t}^{-1} \nabla$ in $\varphi_{t}^{-1} \mathcal{L}$. Then by straightforward calculations we obtain the formula

$$
\rho_{t}([\gamma])^{-1} \frac{d}{d t} \rho_{t}([\gamma])=-\sqrt{-1} \int_{\gamma} \alpha_{V_{t}}
$$

for each $[\gamma] \in \pi_{1}(L)$. Hence the statement of the theorem follows from this formula.

1.1. Moment maps and Lagrangian orbits. We call a Lagrangian submanifold obtained as a Lagrangian orbit of a Lie group a homogeneous Lagrangian submanifold. Suppose that a connected Lie group $K$ acts on $M$ in a Hamiltonian way. Let $\mathfrak{k}$ denote the Lie algebra of Lie group $K$ and $\mathfrak{k}^{*}$ its dual vector space. Let $\mu: M \longrightarrow \mathfrak{k}^{*}$ be the moment map for the Hamiltonian group action $K$ on $M$. By the definition the moment map $\mu$ satisfies the following conditions :

(1) $d\langle\mu, \xi\rangle=\omega(\tilde{\xi}, \cdot)$ for all $\xi \in \mathfrak{k}$.

(2) $\mu(a \cdot x)=\operatorname{Ad}^{*}\left(a^{-1}\right) \mu(x)$ for all $x \in M$ and all $a \in K$.

Here $\tilde{\xi}$ denotes the vector field on $M$ induced by the action of the one-parameter subgroup $\exp (t \xi)$. Set

$$
\mathfrak{z}^{\left(\mathfrak{k}^{*}\right)}:=\left\{\alpha \in \mathfrak{k}^{*} \mid \operatorname{Ad}^{*}(a) \alpha=\alpha \text { for all } a \in K\right\} .
$$

Then we know the following basic properties on moment maps and Lagrangian orbits (see also [7]).

Proposition 1.1. Let $x \in M$. If $K \cdot x$ is an isotropic orbit, then the orbit $K \cdot x$ is contained in a level set $\mu^{-1}(\alpha)$ of the moment map $\mu$ for some $\alpha \in \mathfrak{k}^{*}$, and $\alpha \in \mathfrak{z}\left(\mathfrak{k}^{*}\right)$.

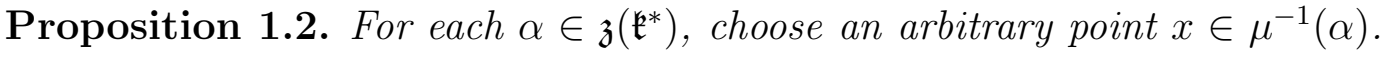
Then the $K$-orbit $K \cdot x$ in $M$ through $x$ has the following properties : 
(1) The orbit $K \cdot x$ of $K$ through $x$ is contained in $\mu^{-1}(\alpha)$.

(2) The orbit $K \cdot x$ is an isotropic submanifold of $M$.

(3) $K \cdot x$ is a Lagrangian submanifold of $M$ if and only if

$$
T_{y}(K \cdot x)=\operatorname{Ker}(d \mu)_{y} \quad \text { for each } y \in K \cdot x,
$$

that is, $K \cdot x$ is an open subset of $\mu^{-1}(\alpha)$.

(4) Assume that the action of $K$ on $M$ is proper. Then $K \cdot x$ is a Lagrangian submanifold of $M$ if and only if the orbit $K \cdot x$ is a connected component of $\mu^{-1}(\alpha)$.

In the case when $K$ is compact, we can identified $\mathfrak{k}^{*}$ with $\mathfrak{k}$ via a fixed $\operatorname{Ad}(K)$ invariant inner product of $\mathfrak{k}$. The following results are on the connectivity of the level subset and the convexity of the image of the moment maps.

Proposition 1.3 ([17], cf. [13]). Suppose that $K$ is a compact, connected Lie group with the Hamiltonian action on a compact symplectic manifold $M$. Let $T$ be a maximal torus of $K$ with Lie algebra $\mathfrak{t}$.

(1) For each $\xi \in \mathfrak{c}(\mathfrak{k}) \cong \mathfrak{z}\left(\mathfrak{k}^{*}\right)$, the level set $\mu^{-1}(\xi)$ of the moment map $\mu$ is a connected subset of $M$.

(2) The intersection $\mu(M) \cap \mathfrak{t}_{+}$of the image of the moment map with the positive Weyl chamber $\mathfrak{t}_{+}$is a compact connected convex subset of $\mathfrak{k} \cong$ $\mathfrak{k}^{*}$.

Problem. Classify homogeneous Lagrangian submanifolds, i.e., Lagrangian orbits of Hamiltonian group actions, in specific symplectic manifolds.

1.2. Hamiltonian minimality and Hamiltonian stability. Now we assume that $(M, \omega, J, g)$ is a Kähler manifold with complex structure $J$ and Kähler metric $g$. Let $\varphi: L \rightarrow M$ be a Lagrangian immersion into $M$. Let $H$ denote the mean curvature vector field of $\varphi$ and we call the corresponding 1-form $\alpha_{H} \in \Omega^{1}(L)$ the mean curvature form of $\varphi$.

The mean curvature form $\alpha_{H}$ must satisfy the following identity ([11]), which follows from the Codazzi equation : $d \alpha_{H}=\varphi^{*} \rho_{M}$, where $\rho_{M}$ denotes the Ricci form of $M$. Thus if $M$ is an Einstein-Kähler manifold, then we have $d \alpha_{H}=0$.

Here we suppose that $L$ is compact without boundary.

Definition 1.1. A Lagrangian immersion $\varphi$ is called Hamiltonian minimal (shortly, H-minimal) or Hamiltonian stationary if under every Hamiltonian deformation $\left\{\varphi_{t}\right\}$ the first variation of the volume vanishes.

By the first variational formula, the H-minimality equation is $\delta \alpha_{H}=0$, where $\delta$ denotes the codifferential operator with respect to the induced metric $\varphi^{*} g$ on $L$. Thus if $M$ is an Einstein-Kähler manifold, then the mean curvature form $\alpha_{H}$ is a harmonic 1-form on $L$.

Definition 1.2. An H-minimal Lagrangian immersion $\varphi$ is called Hamiltonian stable (shortly, H-stable) if under every Hamiltonian deformation $\left\{\varphi_{t}\right\}$ the second variation of the volume is nonnegative. 
The second variational formula is as follows ([31]) :

$$
\begin{aligned}
& \left.\frac{d^{2}}{d t^{2}} \operatorname{Vol}\left(L, \varphi_{t}^{*} g\right)\right|_{t=0} \\
= & \int_{L}\left(\left\langle\Delta_{L}^{1} \alpha, \alpha\right\rangle-\langle\bar{R} \alpha, \alpha\rangle-2\left\langle\alpha \otimes \alpha \otimes \alpha_{H}, S\right\rangle+\left\langle\alpha_{H}, \alpha\right\rangle^{2}\right) d v,
\end{aligned}
$$

where we set $\alpha=\alpha_{V_{0}} \in B_{1}(L)$. Here

$$
\langle\bar{R} \alpha, \alpha\rangle:=\sum_{i, j=1}^{n} \operatorname{Ric}^{M}\left(e_{i}, e_{j}\right) \alpha\left(e_{i}\right) \alpha\left(e_{j}\right),
$$

where $\left\{e_{i}\right\}$ is a local orthonormal frame on $L$ and

$$
S(X, Y, Z):=g(J B(X, Y), Z)=\omega(B(X, Y), Z)
$$

for each $X, Y, Z \in T L$, which is a symmetric 3 -tensor field on $L$ defined by the second fundamental form $B$ of $L$ in $M$.

We know that if a Kähler manifold $M$ is simply connected or $b_{1}(M)=0$, then any holomorphic Killing vector field $V$ on $M$ is always a Hamiltonian vector field, and thus it generates a volume-preserving Hamiltonian deformation of every H-minimal Lagrangian immersion into $M$. Such a Hamiltonian deformation should be considered as a trivial element of the null-space of the second variations.

Definition 1.3. An H-minimal Lagrangian immersion $\varphi$ is called strictly Hamiltonian stable (shortly, strictly H-stable) if $\varphi$ is Hamiltonian stable and the nullspace of the second variations is exactly the span of the normal projections of holomorphic Killing vector fields of the ambient Kähler manifold $M$.

In the case when $L$ is a compact minimal Lagrangian submanifold (i. e. $H \equiv$ $0)$ in an Einstein-Kähler manifold $M$ with Einstein constant $\kappa$, the second variational formula is quite simplified and it follows that $L$ is $\mathrm{H}$-stable if and only if the first (positive) eigenvalue $\lambda_{1}$ of the Laplacian $\Delta_{L}^{0}$ of $L$ on functions satisfies the inequality $\lambda_{1} \geq \kappa$.

We do NOT know so many examples of compact Hamiltonian stable Lagrangian submanifolds yet. The elementary examples of compact H-stable minimal or H-minimal Lagrangian submanifolds are as follows : (1) circles on a plane $S^{1} \subset \mathbf{C}$, (2) great circles and small circles $S^{1} \subset S^{2}=\mathbf{C} P^{1}$, (3) closed circles $S^{1} \subset H^{2}=\mathbf{C} H^{1}$, (4) real projective subspaces $\mathbf{R} P^{n} \subset \mathbf{C} P^{n}$ ([29]), (5) a product of $n+1$ circles $S^{1}\left(r_{0}\right) \times \cdots \times S^{1}\left(r_{n}\right) \subset \mathbf{C}^{n+1}$ and the quotient space by the $S^{1}$-action $T^{n} \subset \mathbf{C} P^{n}([31])$.

In [1], the Hamiltonian stability of compact irreducible minimal Lagrangian submanifolds with parallel second fundamental form (i.e., $\nabla S=0$ ) embedded in complex projective spaces was shown : (a) $S U(p) / S O(p) \mathbf{Z}_{p} \subset \mathbf{C} P^{(p-1)(p+2) / 2}$, (b) $S U(p) / \mathbf{Z}_{p} \subset \mathbf{C} P^{p^{2}-1}$, (c) $S U(2 p) / S p(p) \mathbf{Z}_{2 p} \subset \mathbf{C} P^{(p-1)(2 p+1)}$, (d) $E_{6} / F_{4} \mathbf{Z}_{3} \subset$ $\mathrm{C} P^{26}$.

Lagrangian submanifolds satisfying $\nabla S=0$ are called parallel Lagrangian submanifolds. Parallel Lagrangian submanifolds in complex space forms were classified by H. Naitoh and M. Takeuchi ([25, 28, 26, 27]). Recently the above 
result of [1] is generalized as follows ([2, 3]): If $L$ is a compact parallel Lagrangian submanifold embedded in a complex space form $\left(=\mathbf{C} P^{n}, \mathbf{C}^{n}\right.$ or $\left.\mathbf{C} H^{n}\right)$, then $L$ is Hamiltonian stable.

More recently, an example of a compact Hamiltonian stable minimal Lagrangian submanifold in $\mathbf{C} P^{3}$ with $\nabla S \neq 0$, which is obtained as a minimal Lagrangian $S U(2)$-orbit in $\mathbf{C} P^{3}$, was shown by L. Bedulli and A. Gori ([6]), and independently by [33]: (e) The orbit $\rho_{3}(S U(2))\left[z_{0}^{3}+z_{1}^{3}\right] \subset \mathbf{C} P^{3}$ by the irreducible unitary representation of $S U(2)$ of degree 3 (cf. Section 5 ) is a 3-dimensional compact embedded Hamiltonian stable minimal Lagrangian submanifold with $\nabla S \neq 0$.

L. Bedulli and A. Gori ([5]) characterized the existence of Lagrangian orbits in compact Kähler manifolds with $b^{1,1}(M)=1$ in terms of the Stein property of their complexified orbits. Applying the classification theory of "prehomogeneous vector spaces" due to M. Sato and T. Kimura [38], they classified compact homogeneous Lagrangian submanifolds in $\mathbf{C} P^{n}$ obtained as Lagrangian orbits of compact simple Lie subgroups of $S U(n+1): 16$ examples $=$ [5 examples with $\nabla S=0: \mathbf{R} P^{n}$, (a) $\sim($ d) $]+[11$ examples with $\nabla S \neq 0 \ni$ (e)].

M. Takeuchi (40]) classified all compact totally geodesic Lagrangian submanifolds in compact irreducible Hermitian symmetric spaces. He proved that they all are real forms of Hermitian symmetric spaces, i.e., the fixed point subset of anti-holomorphic isometries, and are given as symmetric R-spaces canonically embedded in compact Hermitian symmetric spaces. The Hamiltonian stability of all compact totally geodesic Lagrangian submanifolds embedded in compact irreducible Hermitian symmetric spaces (with Einstein constant 1/2) are known as follows $([40,[1])$ :

Table 1

\begin{tabular}{|c|c|c|c|c|c|}
\hline$M$ & $L$ & Einstein & $\lambda_{1}$ & H-stable & stable \\
\hline$G_{p, q}(\mathbf{C}), p \leq q$ & $G_{p, q}(\mathbf{R})$ & Yes & $\frac{1}{2}$ & Yes & No \\
\hline$G_{2 p, 2 q}(\mathbf{C}), p \leq q$ & $G_{p, q}(\mathbf{H})$ & Yes & $\frac{1}{2}$ & Yes & Yes \\
\hline$G_{m, m}(\mathbf{C})$ & $U(m)$ & No & $\frac{1}{2}$ & Yes & No \\
\hline$S O(2 m) / U(m)$ & $S O(m), m \geq 5$ & Yes & $\frac{1}{2}$ & Yes & No \\
\hline$S O(4 m) / U(2 m), m \geq 3$ & $U(2 m) / S p(m)$ & No & $\frac{m}{4 m-2}$ & No & No \\
\hline$S p(2 m) / U(2 m)$ & $S p(m), m \geq 2$ & Yes & $\frac{1}{2}$ & Yes & Yes \\
\hline$S p(m) / U(m)$ & $U(m) / O(m)$ & No & $\frac{1}{2}$ & Yes & No \\
\hline$Q_{p+q-2}(\mathbf{C}), q-p \geq 3$ & $Q_{p, q}(\mathbf{R}), p \geq 2$ & No & $\frac{p}{p+q-2}$ & No & No \\
\hline$Q_{p+q-2}(\mathbf{C}), 0 \leq q-p<3$ & $Q_{p, q}(\mathbf{R}), p \geq 2$ & No & $\frac{1}{2}$ & Yes & No \\
\hline$Q_{q-1}(\mathbf{C}), q \geq 3$ & $Q_{1, q}(\mathbf{R})$ & Yes & $\frac{1}{2}$ & Yes & Yes \\
\hline$E_{6} / T \cdot \operatorname{Spin}(10)$ & $P_{2}(\mathbf{K})$ & Yes & $\frac{1}{2}$ & Yes & Yes \\
\hline$E_{6} / T \cdot \operatorname{Spin}(10)$ & $G_{2,2}(\mathbf{H}) / \mathbf{Z}_{2}$ & Yes & $\frac{1}{2}$ & Yes & No \\
\hline$E_{7} / T \cdot E_{6}$ & $S U(8) / S p(4) \mathbf{Z}_{2}$ & Yes & $\frac{1}{2}$ & Yes & No \\
\hline$E_{7} / T \cdot E_{6}$ & $T \cdot E_{6} / F_{4}$ & No & $\frac{1}{6}$ & No & No \\
\hline
\end{tabular}

where $G_{p, q}(\mathbf{F})$ : Grassmanian manifold of all $p$-dimensional subspaces of $\mathbf{F}^{p+q}$, for each $\mathbf{F}=\mathbf{R}, \mathbf{C}, \mathbf{H} . \quad P_{2}(\mathbf{K})$ : Cayley projective plane. $Q_{n}(\mathbf{C})$ : complex 
hyperquadric of complex dimension $n$. Here each $M$ is equipped with the standard Kähler metric of Einstein constant $1 / 2$ and $\lambda_{1}$ denotes the first eigenvalue of the Laplacian of $L$ on smooth functions.

Remark. The second named author apologizes that there are some inaccuracies in the cases of $M=Q_{n}(\mathbf{C})$ at the table of [1, p.608]. It should be corrected as above.

\section{LAGRANGIAN SUBMANIFOLDS IN COMPLEX HYPERQUADRICS AND HYPERSURFACE GEOMETRY IN SPHERES}

Next we shall discuss Lagrangian submanifolds in the complex hyperquadrics

$$
Q_{n}(\mathbf{C}) \cong \widetilde{G r}_{2}\left(\mathbf{R}^{n+2}\right) \cong S O(n+2) / S O(2) \times S O(n),
$$

the latter are compact irreducible Hermitian symmetric spaces of rank 2 if $n \geq 3$ and $S^{2} \times S^{2}$ if $n=2$. Here $Q_{n}(\mathbf{C})$ denotes the complex hypersurface of $\mathbf{C} P^{n+1}$ defined by the algebraic equation $z_{0}^{2}+z_{1}^{2}+\cdots+z_{n+1}^{2}=0$ and $\widetilde{G r}_{2}\left(\mathbf{R}^{n+2}\right)$ denotes the real Grassmann manifold of oriented 2-planes in $\mathbf{R}^{n+2}$. Let $\mathcal{L}$ be the tautological holomorphic line bundle over $Q_{n}(\mathbf{C})$ and through the identification $Q_{n}(\mathbf{C}) \cong \widetilde{G r}_{2}\left(\mathbf{R}^{n+2}\right)$, the holomorphic line bundle $\mathcal{L}$ can be also considered as the tautological real vector bundle $\mathcal{V}$ of rank 2 over $\widetilde{G r}_{2}\left(\mathbf{R}^{n+2}\right)$.

Geometry of Lagrangian submanifolds in complex hyperquadrics has the important relationship with Hypersurface Geometry in the unit sphere $S^{n+1}(1)$. Let $N^{n} \subset S^{n+1}(1) \subset \mathbf{R}^{n+2}$ be an oriented hypersurface immersed in the unit standard sphere. Now we denote by $\vec{x}$ its position vector of point $p$ of $N^{n}$ and by $\vec{n}$ the unit normal vector field of $N^{n}$ in $S^{n+1}(1)$. Then we can define its "Gauss map" by

$$
\mathcal{G}: N^{n} \ni p \longmapsto[\vec{x}(p) \wedge \vec{n}(p)] \cong[\vec{x}(p)+\sqrt{-1} \vec{n}(p)] \in \widetilde{\mathrm{Gr}}_{2}\left(\mathbf{R}^{n+2}\right) \cong Q_{n}(\mathbf{C}) .
$$

Here $[\vec{x}(p) \wedge \vec{n}(p)]$ denotes an oriented 2-plane in $\mathbf{R}^{n+2}$ spanned by two vectors $\vec{x}(p)$ and $\vec{n}(p)$. Then the fundamental fact is that $\mathcal{G}$ is a Lagrangian immersion ([36], [37]). Moreover we can observe that

Proposition 2.1. Let $F: N^{n} \rightarrow S^{n+1}(1)$ be a smooth immersion of an $n$ dimensional oriented smooth manifold $N^{n}$ into the $(n+1)$-dimensional unit sphere $S^{n+1}(1)$ and $\mathcal{G}: N^{n} \rightarrow \widetilde{G r}_{2}\left(\mathbf{R}^{n+2}\right) \cong Q_{n}(\mathbf{C})$ be the Gauss map of $F$.

(1) If $F_{t}: N^{n} \rightarrow S^{n+1}(1)(|t|<c)$ is a smooth family of smooth immersions with $F=F_{0}$, then a smooth family of the Gauss maps $\mathcal{G}_{t}: N^{n} \rightarrow$ $\widetilde{G r}_{2}\left(\mathbf{R}^{n+2}\right) \cong Q_{n}(\mathbf{C})$ of $F_{t}(|t|<c)$ is a Hamiltonian deformation of $\mathcal{G}$.

(2) Suppose that $\varphi_{t}: N^{n} \rightarrow \widetilde{G r}_{2}\left(\mathbf{R}^{n+2}\right) \cong Q_{n}(\mathbf{C})(|t|<c)$ is a Hamiltonian deformation of the Gauss map $\varphi_{0}=\mathcal{G}$. If $N^{n}$ is compact or $\left\{\varphi_{t}\right\}$ is compactly supported, then there exists a positive real number $\delta<c$ and a smooth family of smooth immersions $F_{t}: N^{n} \rightarrow S^{n+1}(1)(|t|<\delta)$ with $F_{0}=F$ such that the Gauss map of $F_{t}$ coincides with $\varphi_{t}$ for each $t$ with $|t|<\delta$.

Proof. (1) For each point $p \in N$, we denote by $\vec{x}_{t}(p)$ the position vector of the point $F(p)$ for the immersion $F_{t}: N^{n} \rightarrow S^{n+1}(1)(|t|<c)$. Let $\vec{n}_{t}$ denote the 
unit normal vector field of the immersion $F_{t}$ compatible with the orientations. Then $\left\{\vec{x}_{t}, \vec{n}_{t}\right\}$ defines an orthonormal frame field of the pull-back vector bundle $\mathcal{G}_{t}^{*} \mathcal{V}$ defined on the whole $N^{n}$, which is parallel with respect to the induced flat connection. Thus for all $t$ the induced flat connections have trivial holonomy, particularly same holonomy. Therefore by Theorem 1.1 the family $\mathcal{G}_{t}: N^{n} \rightarrow$ $Q_{n}(\mathbf{C})(|t|<\delta)$ is a Hamiltonian deformation with $\mathcal{G}_{0}=\mathcal{G}$.

(2) Assume that $\varphi_{t}: N^{n} \rightarrow Q_{n}(\mathbf{C})$ is a Hamiltonian deformation of $\varphi_{0}=\mathcal{G}$. Since the induced flat $U(1)$-connection in $\varphi_{0}^{-1} \mathcal{L}=\mathcal{G}^{-1} \mathcal{L}$ has trivial holonomy, by Theorem 1.1 each induced flat $U(1)$-connection in $\varphi_{t}^{-1} \mathcal{L}$ also has trivial holonomy. Thus for each $t$ we can choose smoothly a parallel orthonormal frame field $\left\{v_{1}^{t}, v_{2}^{t}\right\}$ in $\varphi_{t}^{*} \mathcal{V}$ on $N$ with $\left\{v_{1}^{0}, v_{2}^{0}\right\}=\{\mathbf{x}, \mathbf{n}\}$ at $t=0$. Then a smooth family of smooth maps $F_{t}: N^{n} \ni p \longmapsto v_{1}^{t}(p) \in S^{n+1}(1)$ satisfies $F_{0}=F$ and hence there is a sufficiently small $\delta>0$ such that each $F_{t}(|t|<\delta)$ is an immersion into $S^{n+1}(1)$ whose Gauss map coincides with $\varphi_{t}$.

A local weaker version of Proposition 2.1 was stated also in [37]. B. Palmer ([37]) showed the mean curvature form formula as follows :

$$
\alpha_{H}=d\left(\operatorname{Im}\left(\log \prod_{i=1}^{n}\left(1+\sqrt{-1} \kappa_{i}\right)\right),\right.
$$

where $\kappa_{i}(i=1, \cdots, n)$ denotes the principal curvatures of $N^{n} \subset S^{n+1}(1)$.

In case $n=2$, since $\left(1+\sqrt{-1} \kappa_{1}\right)\left(1+\sqrt{-1} \kappa_{2}\right)=1-K_{N}+\sqrt{-1} H_{N}$, we see that for any minimal surface $N^{2} \subset S^{3}(1)$, its Gauss map $\mathcal{G}: N^{2} \longrightarrow$ $\widetilde{G r}_{2}\left(\mathbf{R}^{4}\right) \cong Q_{2}(\mathbf{C}) \cong S^{2} \times S^{2}$ is a minimal Lagrangian immersion. This case has been investigated by many authors. In the next section we shall discuss the case of general $n$ when all principal curvatures $\kappa_{i}$ are constant.

For a point $[V]$ of $\widetilde{G r}_{2}\left(\mathbf{R}^{n+2}\right)$, the fiber of the vector bundle $\mathcal{V}$ at $[V]$ is given by

$$
(\mathcal{V})_{[V]}=\{([V], v) \mid v \in V\}
$$

where $[V]$ is an oriented 2-dimensional vector subspace of $\mathbf{R}^{n+2}$ considered as a point of $\widetilde{G r}_{2}\left(\mathbf{R}^{n+2}\right)$. For each $[V] \in \widetilde{G r}_{2}\left(\mathbf{R}^{n+2}\right)$, let $V \oplus V^{\perp}=\mathbf{R}^{n+2}$ be the decomposition into $V$ and its orthogonally complementary subspace $V^{\perp}$. We have the identification:

$$
T_{[V]} \widetilde{G r}_{2}\left(\mathbf{R}^{n+2}\right) \cong \operatorname{Hom}\left(V, V^{\perp}\right)
$$

The standard complex structure $\mathcal{J}$ of $\widetilde{G r}_{2}\left(\mathbf{R}^{n+2}\right)$ is defined by

$$
[\mathcal{J}(T)](v):=T(j v)
$$

for each $T \in \operatorname{Hom}\left(V, V^{\perp}\right) \cong T_{[V]} \widetilde{G r}_{2}\left(\mathbf{R}^{n+2}\right)$ and each $v \in V$, where $j$ is a rotation of $\pi / 2$ on the oriented 2-dimensional vector space $V$.

Let $\varphi: L \rightarrow \widetilde{G r}_{2}\left(\mathbf{R}^{n+2}\right)$ be a Lagrangian immersion of an $n$-dimensional connected smooth manifold $L$ and let $g_{L}$ denote a Riemannian metric on $L$ induced by $\varphi$ from the standard Riemannian metric of $\widetilde{G r}_{2}\left(\mathbf{R}^{n+2}\right)$.

Let $\pi_{\varphi}: \varphi^{-1} \mathcal{V} \longrightarrow L$ be the pull-back vector bundle over $L$ with induced flat $U(1)$-connection $\varphi^{-1} \nabla$. For each $\left(p_{0}, v_{0}\right) \in \varphi^{-1} \mathcal{V}$, where $\left(\left[V_{0}\right], v_{0}\right) \in \mathcal{V}_{\varphi\left(p_{0}\right)}$

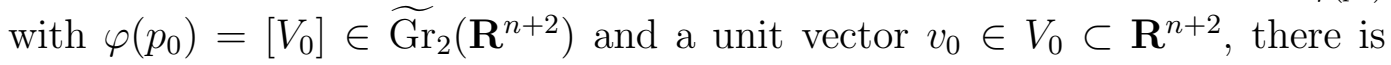


a unique maximal connected integral manifold $\tilde{N}$ through $\left(p_{0}, v_{0}\right)$ in $\varphi^{-1} \mathcal{V}$ of the horizontal distribution with respect to the flat connection $\varphi^{-1} \nabla$. Then $\pi_{\varphi}: \tilde{N} \rightarrow L$ is a smooth covering map with the deck transformation group $\rho\left(\pi_{1}(L)\right) \subset U(1)$, where $\rho: \pi_{1}(L) \rightarrow U(1)$ is the holonomy homomorphism of the flat connection $\varphi^{-1} \nabla$. We define a smooth map $F$ by

$$
F: \tilde{N} \ni(x, v) \longmapsto v \in S^{n+1}(1) .
$$

We should note that the map $F$ is not always an immersion and thus the image $F(\tilde{N})$ does not necessarily give a hypersurface in $S^{n+1}(1)$.

We express $\varphi$ as

$$
\varphi: L \ni p \longmapsto \varphi(p)=\left[V_{p}\right] \in \widetilde{\mathrm{Gr}}_{2}\left(\mathbf{R}^{n+2}\right),
$$

where $V_{p}$ is an oriented 2-dimensional vector subspace of $\mathbf{R}^{n+2}$ representing the point $\varphi(p)$. The differential of $\varphi$

$$
(d \varphi)_{p}: T_{p} L \longrightarrow T_{\left[V_{p}\right]} \widetilde{G r}_{2}\left(\mathbf{R}^{n+2}\right) \cong \operatorname{Hom}\left(V_{p}, V_{p}^{\perp}\right)
$$

is an injective isometric linear map. For each vector $v \in V_{p}$, we introduce a linear operator

defined by

$$
B_{v}: T_{p} L \rightarrow V_{p}^{\perp} \subset \mathbf{R}^{n+2}
$$

for each $X \in T_{p} L$.

$$
B_{v}(X):=\left[(d \varphi)_{p}(X)\right](v)
$$

Proposition 2.2. The linear operators $B_{v}\left(v \in V_{p}\right)$ have the following properties :

$$
{ }^{t} B_{v} \circ B_{j v}={ }^{t} B_{j v} \circ B_{v} \quad \text { for each } v \in V_{p}
$$

and

$$
{ }^{t} B_{v} \circ B_{v}+{ }^{t} B_{j v} \circ B_{j v}=\text { Id } \quad \text { for each } v \in V_{p} .
$$

Proof. It follows from the Lagrangian condition of $\varphi$ that

$$
\begin{aligned}
& \left\langle\left[(d \varphi)_{p}(X)\right]\left(j v_{1}\right),\left[(d \varphi)_{p}(Y)\right]\left(v_{1}\right)\right\rangle+\left\langle\left[(d \varphi)_{p}(X)\right]\left(j v_{2}\right),\left[(d \varphi)_{p}(Y)\right]\left(v_{2}\right)\right\rangle \\
= & \left\langle\left[(d \varphi)_{p}(X)\right]\left(j v_{1}\right),\left[(d \varphi)_{p}(Y)\right]\left(v_{1}\right)\right\rangle-\left\langle\left[(d \varphi)_{p}(X)\right]\left(v_{1}\right),\left[(d \varphi)_{p}(Y)\right]\left(j v_{1}\right)\right\rangle \\
= & 0
\end{aligned}
$$

for each $X, Y \in T_{p} L$, where $\left\{v_{1}, v_{2}=j v_{1}\right\}$ is an orthonormal basis of $V_{p}$ compatible with its orientation. Thus

$$
\left\langle B_{j v_{1}}(X), B_{v_{1}}(Y)\right\rangle=\left\langle B_{v_{1}}(X), B_{j v_{1}}(Y)\right\rangle
$$

for each $X, Y \in T_{p} L$. The condition (2.5) is equal to

$$
{ }^{t} B_{v_{1}} \circ B_{j v_{1}}={ }^{t} B_{j v_{1}} \circ B_{v_{1}} .
$$

On the other hand, the isometric condition of $\varphi$ implies

$$
\begin{aligned}
& \left\langle\left[(d \varphi)_{p}(X)\right]\left(v_{1}\right),\left[(d \varphi)_{p}(Y)\right]\left(v_{1}\right)\right\rangle+\left\langle\left[(d \varphi)_{p}(X)\right]\left(j v_{1}\right),\left[(d \varphi)_{p}(Y)\right]\left(j v_{1}\right)\right\rangle \\
= & \langle X, Y\rangle
\end{aligned}
$$

for each $X, Y \in T_{p} L$. So we have

$$
{ }^{t} B_{v_{1}} \circ B_{v_{1}}+{ }^{t} B_{j v_{1}} \circ B_{j v_{1}}=\mathrm{Id} .
$$


Moreover, by using (2.3) and (2.4) we get

Lemma 2.1. For any $v=\cos \theta \cdot v_{1}+\sin \theta \cdot j v_{1} \in V_{p}$,

$$
{ }^{t} B_{v} \circ B_{v}=\cos 2 \theta\left({ }^{t} B_{v_{1}} \circ B_{v_{1}}\right)+\sin 2 \theta\left({ }^{t} B_{j v_{1}} \circ B_{v_{1}}\right)+\sin ^{2} \theta \mathrm{I} .
$$

If we suppose that $0 \neq X \in \operatorname{Ker} B_{v_{1}} \subset T_{p} L$, then we have

$$
\begin{aligned}
\left({ }^{t} B_{v} \circ B_{v}\right) X & =\cos 2 \theta\left({ }^{t} B_{v_{1}} \circ B_{v_{1}}\right) X+\sin 2 \theta\left({ }^{t} B_{j v_{1}} \circ B_{v_{1}}\right) X+\sin ^{2} \theta X \\
& =\sin ^{2} \theta X .
\end{aligned}
$$

Thus we see that if $0<\theta<\pi$, then $X \notin \operatorname{Ker} B_{v}$. Note that $\left\|B_{v}(X)\right\|=$ $|\sin \theta| \cdot\|X\|$. More strongly we obtain

Lemma 2.2. $\operatorname{Ker} B_{v} \perp \operatorname{Ker} B_{v_{1}}$ for each $\theta$ with $0<\theta<\pi$.

Proof. For each $X \in \operatorname{Ker} B_{v}$ and each $Y \in \operatorname{Ker} B_{v_{1}}$, we have

$$
\begin{aligned}
0 & =\left\langle\left({ }^{t} B_{v} \circ B_{v}\right) X, Y\right\rangle \\
& =\cos 2 \theta\left\langle\left({ }^{t} B_{v_{1}} \circ B_{v_{1}}\right) X, Y\right\rangle+\sin 2 \theta\left\langle\left({ }^{t} B_{j v_{1}} \circ B_{v_{1}}\right) X, Y\right\rangle+\sin ^{2} \theta\langle X, Y\rangle \\
& =\cos 2 \theta\left\langle\left({ }^{t} B_{v_{1}} \circ B_{v_{1}}\right) X, Y\right\rangle+\sin 2 \theta\left\langle\left({ }^{t} B_{v_{1}} \circ B_{j v_{1}}\right) X, Y\right\rangle+\sin ^{2} \theta\langle X, Y\rangle \\
& =\cos 2 \theta\left\langle B_{v_{1}} X, B_{v_{1}} Y\right\rangle+\sin 2 \theta\left\langle B_{j v_{1}} X, B_{v_{1}} Y\right\rangle+\sin ^{2} \theta\langle X, Y\rangle \\
& =\sin ^{2} \theta\langle X, Y\rangle .
\end{aligned}
$$

Therefore we obtain

Lemma 2.3. Except for finitely many $v \in V_{p} \cap S^{n+1}(1), B_{v}: T_{p} L \rightarrow V_{p}{ }^{\perp}$ is a linear isomorphism.

For such a vector $v$, there is a unique maximal connected integral manifold $\tilde{N}$ through $(p, v)$ in $\varphi^{-1} \mathcal{V}$ of the horizontal distribution with respect to the flat connection $\varphi^{-1} \nabla$. The map $F: \tilde{N} \ni(x, w) \longmapsto w \in S^{n+1}(1)$ is an immersion in a neighborhood of $(p, v)$ and $F(\tilde{N})$ gives an immersed oriented hypersurface $S^{n+1}(1)$ around $(p, v)$.

\section{LAGRANGIAN SUBMANIFOLDS IN COMPLEX HYPERQUADRICS OBTAINED AS Gauss ImAges of ISOPARAMETRIC HYPERSURFACES IN SPHERES}

Now suppose that $N^{n}$ is a compact oriented hypersurface in $S^{n+1}(1)$ with constant principal curvatures, the so called "isoparametric hypersurface". By Münzner's result ([21],22]), $N^{n}$ is real algebraic in the sense that it is defined by a certain homogeneous real algebraic equation (Cartan-Münzner polynomial) and the number $g$ of distinct principal curvatures must be $g=1,2,3,4$ or 6 . Then the "Gauss image" of a minimal Lagrangian immersion $\mathcal{G}: N^{n} \rightarrow Q_{n}(\mathbf{C})$ is a compact minimal Lagrangian submanifold $L=\mathcal{G}\left(N^{n}\right)=N^{n} / \mathbf{Z}_{g}$ embedded in $Q_{n}(\mathbf{C})$ obtained as the quotient space of $N^{n}$ by a free action of a finite cyclic group $\mathbf{Z}_{g}$ of order $g$. We remark that $g=1$ or 2 if and only if $\mathcal{G}: N^{n} \rightarrow Q_{n}(\mathbf{C})$ is a totally geodesic Lagrangian immersion. All isoparametric hypersurfaces in spheres are classified into homogeneous ones, which are given as principal orbits of compact group actions on spheres with cohomogeneity 1, and nonhomogneous ones, which were discovered first by H. Ozeki-M. Takeuchi ([34], 
[35]), and developed by D. Ferus-H. Karcher-H. F. Münzner ([12]) and recently T. Cecil-Q.-S. Chi-G. R. Jensen ([9]). Concerned with the homogeneity, we can observe

Proposition 3.1. An isoparametric hypersurface $N^{n} \subset S^{n+1}(1)$ is homogeneous, i.e., an orbit of a compact connected Lie subgroup $K \subset S O(n+2)$, if and only if its Gauss image $\mathcal{G}\left(N^{n}\right)$ is a homogeneous Lagrangian submanifold in $Q_{n}(\mathbf{C})$.

The part of "only if "is trivial. Here we give a proof for the part of "if ". Assume that $\mathcal{G}\left(N^{n}\right)$ is homogeneous, that is, a Lagrangian orbit $\mathcal{G}\left(N^{n}\right)=$ $K \cdot\left[V_{0}\right]$ through a point $\left[V_{0}\right]$ of a compact connected Lie subgroup $K$ of $S O(n+$ 2 ). Then $g_{L}$ is a $K$-invariant Riemannian metric on $L$. In order to prove that $N$ is also homogeneous, we analyze the $K$-equivariance of the bundle homomorphism

$$
B: \mathcal{V} \otimes T L \ni v \otimes X \longmapsto B_{v}(X) \in \mathcal{V}^{\perp}
$$

For $p \in N$, let $\left[V_{\mathcal{G}(p)}\right]=\mathcal{G}(p)$ denote an oriented 2-dimensional vector subspace of $\mathbf{R}^{n+2}$ spanned by $\mathbf{x}(p)$ and $\mathbf{n}(p)$ with the orientation determined by $\{\mathbf{x}(p), \mathbf{n}(p)\}$.

Let $\left\{\mathbf{e}_{1}, \cdots, \mathbf{e}_{n}\right\}$ be a local orthonormal frame field compatible with the orientation of $N$ around $p$ such that $h\left(\mathbf{e}_{i}, \mathbf{e}_{j}\right)=\kappa_{i} \delta_{i j}(i, j=1, \cdots, n)$, or equivalently $A\left(\mathbf{e}_{i}\right)=\kappa_{i} \mathbf{e}_{i}(i=1, \cdots, n)$, where $h$ and $A$ denote the second fundamental form and the shape operator or the Weingarten map of the hypersurface $N^{n}$ in $S^{n+1}(1)$, respectively. We denote by $\left\{k_{1}, \cdots, k_{g}\right\}$ the distinct principal curvatures of $N^{n}$ in $S^{n+1}(1)$.

For each $X \in T_{p} N$ and each $v=\cos \phi \mathbf{x}(p)+\sin \phi \mathbf{n}(p) \in V_{\mathcal{G}(p)}$,

$$
B_{v}\left((d \mathcal{G})_{p}(X)\right)=\left[(d \mathcal{G})_{p}(X)\right](v)=\cos \phi X-\sin \phi A(X) .
$$

Since

$$
B_{v}\left((d \mathcal{G})_{p}\left(\mathbf{e}_{i}\right)\right)=\left(\cos \phi-\kappa_{i} \sin \phi\right) \mathbf{e}_{i} \quad(i=1, \cdots, n),
$$

the linear endomorphism ${ }^{t} B_{v} \circ B_{v}: T_{\mathcal{G}(p)} L \rightarrow T_{\mathcal{G}(p)} L$ satisfies

$$
\left({ }^{t} B_{v} \circ B_{v}\right)\left((d \mathcal{G})_{p}\left(\mathbf{e}_{i}\right)\right)=\frac{\left(\cos \phi-\kappa_{i} \sin \phi\right)^{2}}{1+\kappa_{i}^{2}}(d \mathcal{G})_{p}\left(\mathbf{e}_{i}\right) \quad(i=1, \cdots, n)
$$

Note that

$$
\frac{\left(\cos \phi-\kappa_{i} \sin \phi\right)^{2}}{1+\kappa_{i}^{2}}=\frac{\left(\cos \phi-\kappa_{j} \sin \phi\right)^{2}}{1+\kappa_{j}^{2}}
$$

for all $\phi \in \mathbf{R}$ if and only if $\kappa_{i}=\kappa_{j}$. The eigenspace decomposition of $A$ at $p$

$$
T_{p} N=\left(E_{1}\right)_{p} \oplus \cdots \oplus\left(E_{g}\right)_{p}
$$

corresponds the eigenspace decomposition of the symmetric linear endomorphism ${ }^{t} B_{v} \circ B_{v}$ at $\mathcal{G}(p)$

$$
T_{\mathcal{G}(p)} L=\left(E_{1}^{\prime}\right)_{\mathcal{G}(p)} \oplus \cdots \oplus\left(E_{g}^{\prime}\right)_{\mathcal{G}(p)}
$$

via the linear isomorphism $(d \mathcal{G})_{p}: T_{p} N \longrightarrow T_{\mathcal{G}(p)} L$. Then on each $\left(E_{i}^{\prime}\right)_{\mathcal{G}(p)}$ we have

$$
{ }^{t} B_{v} \circ B_{v}=\frac{\left(\cos \phi-k_{i} \sin \phi\right)^{2}}{1+k_{i}^{2}} \operatorname{Id}_{\left(E_{i}^{\prime}\right)_{\mathcal{G}(p)}} .
$$


Lemma 3.1. Let $p_{0}, p \in N^{n}$ and $a \in K \subset S O(n+2)$. Assume that $\mathcal{G}(p)=$ $a \mathcal{G}\left(p_{0}\right)$. Then we have

$$
(\mathbf{x}(p), \mathbf{n}(p))= \pm\left(a \mathbf{x}\left(p_{0}\right), a \mathbf{n}\left(p_{0}\right)\right)\left(\begin{array}{cc}
\cos (m \pi / g) & -\sin (m \pi / g) \\
\sin (m \pi / g) & \cos (m \pi / g)
\end{array}\right) .
$$

for some integer $m$.

Proof. For arbitrary $v_{0} \in V_{\mathcal{G}\left(p_{0}\right)}$, we set $v=a v_{0} \in V_{\mathcal{G}(p)} \subset \mathbf{R}^{n+2}$. We express $v_{0}$ as $v_{0}=(\cos \theta) \mathbf{x}\left(p_{0}\right)+(\sin \theta) \mathbf{n}\left(p_{0}\right)$, and we also express $v$ as $v=(\cos \phi) \mathbf{x}(p)+$ $(\sin \phi) \mathbf{n}(p)$. Since $v=a v_{0}=(\cos \theta) a \mathbf{x}\left(p_{0}\right)+(\sin \theta) a \mathbf{n}\left(p_{0}\right)$, setting

$$
(\mathbf{x}(p), \mathbf{n}(p))=\left(a \mathbf{x}\left(p_{0}\right), a \mathbf{n}\left(p_{0}\right)\right)\left(\begin{array}{cc}
\cos \psi & -\sin \psi \\
\sin \psi & \cos \psi
\end{array}\right)
$$

for some $\psi \in \mathbf{R}$, we get $\phi+\psi \equiv \theta \bmod 2 \pi$. By the $K$-equivariance we have

$$
\left({ }^{t} B_{v} \circ B_{v}\right) \circ(d a)_{\mathcal{G}\left(p_{0}\right)}=(d a)_{\mathcal{G}\left(p_{0}\right)} \circ\left({ }^{t} B_{v_{0}} \circ B_{v_{0}}\right) .
$$

Through a linear isomorphism $(d a)_{\mathcal{G}\left(p_{0}\right)}: T_{\mathcal{G}\left(p_{0}\right)} L \rightarrow T_{\mathcal{G}\left(p_{0}\right)} L$, the direct sum decomposition

$$
T_{\mathcal{G}\left(p_{0}\right)} L=\left(E_{1}^{\prime}\right)_{\mathcal{G}\left(p_{0}\right)} \oplus \cdots \oplus\left(E_{g}^{\prime}\right)_{\mathcal{G}\left(p_{0}\right)}
$$

is mapped to a direct sum decomposition

$$
T_{\mathcal{G}(p)} L=(d a)_{\mathcal{G}\left(p_{0}\right)}\left(E_{1}^{\prime}\right)_{\mathcal{G}\left(p_{0}\right)} \oplus \cdots \oplus(d a)_{\mathcal{G}\left(p_{0}\right)}\left(E_{g}^{\prime}\right)_{\mathcal{G}\left(p_{0}\right)}
$$

Thus by (3.1) on each $(d a)_{\mathcal{G}\left(p_{0}\right)}\left(E_{j}^{\prime}\right)_{\mathcal{G}\left(p_{0}\right)}$ we have

$$
{ }^{t} B_{v} \circ B_{v}=\frac{\left(\cos \theta-k_{j} \sin \theta\right)^{2}}{1+k_{j}^{2}} \operatorname{Id}_{(d a)_{\mathcal{G}\left(p_{0}\right)}}\left(E_{j}^{\prime}\right)_{\mathcal{G}\left(p_{0}\right)} .
$$

Hence for each $i(=1,2, \cdots, g)$, there is uniquely $j(=1,2, \cdots, g)$ such that

$$
\left(E_{i}^{\prime}\right)_{\mathcal{G}(p)}=(d a)_{\mathcal{G}\left(p_{0}\right)}\left(E_{j}^{\prime}\right)_{\mathcal{G}\left(p_{0}\right)}
$$

Since we have

$$
\begin{aligned}
\frac{\left(\cos \theta-k_{j} \sin \theta\right)^{2}}{1+k_{j}^{2}} & =\frac{\left(\cos \phi-k_{i} \sin \phi\right)^{2}}{1+k_{i}^{2}} \\
& =\frac{\left(\cos (\theta-\psi)-k_{i} \sin (\theta-\psi)\right)^{2}}{1+k_{i}^{2}}
\end{aligned}
$$

for all $\theta \in \mathbf{R}$, it implies the equation $\left(1+k_{i} k_{j}\right) \sin \psi=\left(k_{i}-k_{j}\right) \cos \psi$ for each $i$. Suppose that $\sin \psi \neq 0$. Then $k_{i}-k_{j} \neq 0$ and thus it becomes

$$
\cot \psi=\frac{1+k_{i} k_{j}}{k_{i}-k_{j}}=\cot \left(\beta_{i}-\beta_{j}\right) \quad \text { for all } i \text {. }
$$

Here set $k_{i}=\cot \beta_{i}\left(0<\beta_{i}<\pi\right)$. Since we know from the theory of isoparametric hypersurfaces $([21])$ that $\beta_{i}-\beta_{j} \in(\pi / g) \mathbf{Z}$, we obtain that $\psi \in(\pi / g) \mathbf{Z}+\pi \mathbf{Z}$.

By the continuation argument on $N^{n}$ and $K$, it follows from Lemma 3.1 that $N^{n}=K \cdot p_{0}$ and thus $N^{n}$ is homogeneous. We complete the proof of Proposition 3.1 
By virtue of the results of Hsiang-Lawson ([16]), Takagi-Takahashi ([39]), we know that all homogeneous isoparametric hypersurfaces $N^{n} \subset S^{n+1}(1)$ can be obtained as principal orbits of compact Riemannian symmetric pairs $(U, K)$ of rank 2 :

Table 2

\begin{tabular}{|c|c|c|c|c|c|}
\hline$g$ & Type & $(U, K)$ & $\operatorname{dim} N$ & $m_{1}, m_{2}$ & $N=K / K_{0}$ \\
\hline 1 & $\begin{array}{c}S^{1} \times \\
\text { BDII }\end{array}$ & $\begin{array}{c}\left(S^{1} \times S O(n+2), S O(n+1)\right) \\
(n \geq 1)\end{array}$ & $n$ & $n$ & $S^{n}$ \\
\hline 2 & $\begin{array}{c}\mathrm{BDII} \\
\times \mathrm{BDII}\end{array}$ & $\begin{array}{c}(S O(p+2) \times S O(n+2-p) \\
S O(p+1) \times S O(n+1-p)) \\
(1 \leq p \leq n-1)\end{array}$ & $n$ & $p, n-p$ & $S^{p} \times S^{n-p}$ \\
\hline 3 & $\mathrm{AI}_{2}$ & $(S U(3), S O(3))$ & 3 & 1,1 & $\frac{S O(3)}{\mathbf{Z}_{2}+\mathbf{Z}_{2}}$ \\
\hline 3 & $\mathfrak{a}_{2}$ & $(S U(3) \times S U(3), S U(3))$ & 6 & 2,2 & $\frac{S U(3)}{T^{2}}$ \\
\hline 3 & $\overline{\mathrm{AII}_{2}}$ & $(S U(6), S p(3))$ & 12 & 4,4 & $\frac{S p(3)}{S p(1)^{3}}$ \\
\hline 3 & EIV & $\left(E_{6}, F_{4}\right)$ & 24 & 8,8 & $\frac{F_{4}}{\operatorname{Spin}(8)}$ \\
\hline 4 & $\mathfrak{b}_{2}$ & $(S O(5) \times S O(5), S O(5))$ & 8 & 2,2 & $\frac{S O(5)}{T^{2}}$ \\
\hline 4 & $\mathrm{AIII}_{2}$ & $\begin{array}{c}(S U(m+2), S(U(m) \times U(2))) \\
(m \geq 2)\end{array}$ & $4 m-2$ & $\begin{array}{c}2, \\
2 m-3\end{array}$ & $\frac{S(U(m) \times U(2))}{S U(m-2) \times T^{2}}$ \\
\hline 4 & $\mathrm{BDI}_{2}$ & $\begin{array}{c}(S O(m+2), S O(m) \times S O(2)) \\
(m \geq 3)\end{array}$ & $2 m-2$ & $\begin{array}{c}1, \\
m-2\end{array}$ & $\frac{S O(m) \times S O(2)}{S O(m-2) \times \mathbf{Z}_{2}}$ \\
\hline 4 & $\mathrm{CII}_{2}$ & $\begin{array}{c}(S p(m+2), S p(m) \times S p(2)) \\
(m \geq 2)\end{array}$ & $8 m-2$ & $\begin{array}{c}4, \\
4 m-5\end{array}$ & $\frac{S p(m) \times S p(2)}{S p(m-2) \times S p(1)^{2}}$ \\
\hline 4 & $\mathrm{DIII}_{2}$ & $(S O(10), U(5))$ & 18 & 4,5 & $\frac{U(5)}{\frac{U U(2) \times S U(2) \times T^{1}}{S}}$ \\
\hline 4 & EIII & $\left(E_{6}, \operatorname{Spin}(10) \cdot T\right)$ & 30 & 6,9 & $\frac{\operatorname{Spin}(10) \cdot T}{S U(4) \cdot T}$ \\
\hline 6 & $\mathfrak{g}_{2}$ & $\left(G_{2} \times G_{2}, G_{2}\right)$ & 12 & 2,2 & $\frac{G_{2}}{T^{2}}$ \\
\hline 6 & G & $\left(G_{2}, S O(4)\right)$ & 6 & 1,1 & $\frac{S O(4)}{\mathbf{Z}_{2}+\mathbf{Z}_{2}}$ \\
\hline
\end{tabular}

Here $m_{1}, m_{2}$ denote the multiplicities of the principal curvatures of $N^{n}$. Let $\mathfrak{u}=\mathfrak{k}+\mathfrak{p}$ be the canonical decomposition of $\mathfrak{u}$ as a symmetric Lie algebra of a symmetric pair $(U, K)$ and $\mathfrak{a}$ be a maximal abelian subspace of $\mathfrak{p}$. Let $B_{\mathfrak{u}}($,$) denote the Killing-Cartan form of the Lie algebra \mathfrak{u}$ if $(U, K)$ is not of type $S^{1} \times$ BDII, or the direct sum of the $(-1)$-times standard inner product of $\sqrt{-1} \mathbf{R}$ and the Killing-Cartan form of $\mathfrak{u}$ if $(U, K)$ is of type $S^{1} \times$ BDII. Define the $\operatorname{Ad}(U)$-invariant inner product $\langle,\rangle_{\mathfrak{u}}$ of $\mathfrak{u}$ by $\langle X, Y\rangle_{\mathfrak{u}}:=-B_{\mathfrak{u}}(X, Y)$ for each $X, Y \in \mathfrak{u}$. The vector space $\mathfrak{p}$ is identified with the Euclidean space $\mathbf{R}^{n+2}$ with respect to the inner product $\langle,\rangle_{\mathfrak{u}}$. The $(n+1)$-dimensional unit sphere $S^{n+1}(1)$ in $\mathfrak{p}$ is defined as

$$
S^{n+1}(1):=\left\{X \in \mathfrak{p} \mid\|X\|_{\mathfrak{u}}^{2}=\langle X, X\rangle_{\mathfrak{u}}=1\right\}
$$

The isotropy linear action $\operatorname{Ad}_{\mathfrak{p}}$ of $K$ on $\mathfrak{p}$ and thus $S^{n+1}(1)$ induces the group action of $K$ on $\widetilde{G r}_{2}(\mathfrak{p}) \cong Q_{n}(\mathbf{C})$. For each regular element $H$ of $\mathfrak{a} \cap S^{n+1}(1)$, 
we get a homogeneous isoparametric hypersurface in the unit sphere

$$
N^{n}=\left(\operatorname{Ad}_{\mathfrak{p}} K\right) H \subset S^{n+1}(1) \subset \mathbf{R}^{n+2} \cong \mathfrak{p} .
$$

Its Gauss image is

$$
\mathcal{G}\left(N^{n}\right)=K \cdot[\mathfrak{a}]=\left[\left(\operatorname{Ad}_{\mathfrak{p}} K\right) \mathfrak{a}\right] \subset \widetilde{G r}_{2}(\mathfrak{p}) \cong Q_{n}(\mathbf{C}) .
$$

Here $N$ and $\mathcal{G}\left(N^{n}\right)$ have homogeneous space expressions $N \cong K / K_{0}$ and $\mathcal{G}\left(N^{n}\right) \cong K / K_{[\mathfrak{a}]}$, where we set

$$
\begin{aligned}
K_{0} & :=\left\{k \in K \mid \operatorname{Ad}_{\mathfrak{p}}(k)(H)=H\right\} \\
& =\left\{k \in K \mid \operatorname{Ad}_{\mathfrak{p}}(k)(H)=H \text { for each } H \in \mathfrak{a}\right\}, \\
K_{\mathfrak{a}} & :=\left\{k \in K \mid \operatorname{Ad}_{\mathfrak{p}}(k)(\mathfrak{a})=\mathfrak{a}\right\}, \\
K_{[\mathfrak{a}]} & :=\left\{k \in K_{\mathfrak{a}} \mid \operatorname{Ad}_{\mathfrak{p}}(k): \mathfrak{a} \longrightarrow \mathfrak{a} \text { preserves the orientation of } \mathfrak{a}\right\} .
\end{aligned}
$$

The deck transformation group of the covering map $\mathcal{G}: N \rightarrow \mathcal{G}\left(N^{n}\right)$ is equal to $K_{[\mathfrak{a}]} / K_{0}=W(U, K) / \mathbf{Z}_{2} \cong \mathbf{Z}_{g}$, where $W(U, K)=K_{\mathfrak{a}} / K_{0}$ is the Weyl group of $(U, K)$.

The moment map $\mu$ of the action of $K$ on $Q_{n}(\mathbf{C})$ induced by the adjoint action of $K$ on $\mathfrak{p}$ is given as follows :

$$
\mu: Q_{n}(\mathbf{C}) \cong \widetilde{G r}_{2}(\mathfrak{p}) \ni[\mathbf{a}+\sqrt{-1} \mathbf{b}]=[V] \longmapsto-[\mathbf{a}, \mathbf{b}] \in \mathfrak{k} \cong \mathfrak{k}^{*}
$$

where $\{\mathbf{a}, \mathbf{b}\}$ is an orthonormal basis of $V \subset \mathfrak{p}$ compatible with its orientation. Then we obtain

$$
\mathcal{G}\left(N^{n}\right)=\mu^{-1}(0) .
$$

For each $[V] \in \widetilde{G r}_{2}(\mathfrak{p})$, the square norm of the moment map

$$
\|\mu([V])\|^{2}=\|[\mathbf{a}, \mathbf{b}]\|^{2}
$$

is equal to the sectional curvature of compact symmetric space $\left(U / K, g_{U}\right)$ for a 2-plane $V$ (cf. [15], [18]). Here $g_{U}$ denotes the invariant Riemannian metric on $U / K$ induced by the $\operatorname{Ad}(U)$-invariant inner product $\langle,\rangle_{\mathfrak{u}}$ of $\mathfrak{u}$.

Let $\Sigma(U, K)$ be the set of (restricted) roots of $(\mathfrak{u}, \mathfrak{k})$ and $\Sigma^{+}(U, K)$ be its subset of positive roots. Note that each $\gamma \in \Sigma(U, K)$ is an $\mathbf{R}$-linear function $\gamma: \mathfrak{a} \rightarrow \sqrt{-1} \mathbf{R}$. We have the root decomposition of $\mathfrak{k}$ and $\mathfrak{p}$ as follows (cf. [39]):

$$
\mathfrak{k}=\mathfrak{k}_{0}+\sum_{\gamma \in \Sigma^{+}(U, K)} \mathfrak{k}_{\gamma}, \quad \mathfrak{p}=\mathfrak{a}+\sum_{\gamma \in \Sigma^{+}(U, K)} \mathfrak{p}_{\gamma},
$$

where

$$
\begin{aligned}
\mathfrak{k}_{0} & :=\{X \in \mathfrak{k} \mid[X, \mathfrak{a}] \subset \mathfrak{a}\} \\
& =\{X \in \mathfrak{k} \mid[X, H]=0 \quad \text { for each } H \in \mathfrak{a}\}, \\
\mathfrak{k}_{\gamma}: & =\left\{X \in \mathfrak{k} \mid(\operatorname{ad} H)^{2} X=(\gamma(H))^{2} X \text { for each } H \in \mathfrak{a}\right\}, \\
\mathfrak{p}_{\gamma} & :=\left\{Y \in \mathfrak{p} \mid(\operatorname{ad} H)^{2} Y=(\gamma(H))^{2} Y \text { for each } H \in \mathfrak{a}\right\} .
\end{aligned}
$$

For each $\gamma \in \Sigma^{+}(U, K)$, set $m(\gamma):=\operatorname{dim} \mathfrak{k}_{\gamma}=\operatorname{dim} \mathfrak{p}_{\gamma}$. Define

$$
\mathfrak{m}:=\sum_{\gamma \in \Sigma^{+}(U, K)} \mathfrak{k}_{\gamma} \quad \text { and } \quad \mathfrak{a}^{\perp}:=\sum_{\gamma \in \Sigma^{+}(U, K)} \mathfrak{p}_{\gamma} .
$$


We can choose orthonormal bases of $\mathfrak{m}$ and $\mathfrak{a}^{\perp}$ with respect to $\langle,\rangle_{\mathfrak{u}}$

$$
\left\{X_{\gamma, i} \mid \gamma \in \Sigma^{+}(U, K), i=1,2, \cdots, m(\gamma)\right\}
$$

and

$$
\left\{Y_{\gamma, i} \mid \gamma \in \Sigma^{+}(U, K), i=1,2, \cdots, m(\gamma)\right\}
$$

such that

$$
\left[H, X_{\gamma, i}\right]=\sqrt{-1} \gamma(H) Y_{\gamma, i}, \quad\left[H, Y_{\gamma, i}\right]=-\sqrt{-1} \gamma(H) X_{\gamma, i}
$$

for each $H \in \mathfrak{a}$.

The following condition (3.10) is used in the classification of Lagrangian orbits in complex hyperquadrics in the next section.

Lemma 3.2. Assume that $(U, K)$ is a compact Riemannian symmetric pair in Table 2. Then $(U, K)$ satisfies the condition

$$
\{0\} \neq \mathfrak{c}(\mathfrak{k}) \subset \mathfrak{m}
$$

if and only if $(U, K)$ is one of the following pairs :

(a) $\left(S^{1} \times S O(3), S O(2)\right)\left[S^{1} \times\right.$ BDII, $\left.n=1\right]$.

(b) $(S O(3) \times S O(3), S O(2) \times S O(2))$ [BDII $\times$ BDII, $n=2]$.

(c) $(S O(3) \times S O(n+1), S O(2) \times S O(n))(n \geq 3)[\mathrm{BDII} \times \mathrm{BDII}, p=1$ or $n-$ $1]$.

(d) $(S O(m+2), S O(2) \times S O(m))(n=2 m-2, m \geq 3)\left[\mathrm{BDI}_{2}\right]$.

We shall prove Lemma 3.2. According to Table 2, if $K$ has the nontrivial center, then $(U, K)$ must be $\left(S^{1} \times S O(3), S O(2)\right),(S O(3) \times S O(n+1), S O(2) \times$ $S O(n))(n \geq 2)$ or an irreducible Hermitian symmetric pair $(U, K)$ of compact type and of rank 2. It is obvious that $(U, K)=\left(S^{1} \times S O(3), S O(2)\right)$ and $(U, K)=(S O(3) \times S O(n+1), S O(2) \times S O(n))(n \geq 2)$ satisfy the condition (3.10). Now we assume that $(U, K)$ is an irreducible Hermitian symmetric pair of compact type and of rank 2 . Note that $\operatorname{dim} \mathfrak{c}(\mathfrak{k})=1$. We refer [15] for results from the theory of Hermitian symmetric Lie algebras. Let $\mathfrak{u}=\mathfrak{k}+\mathfrak{p}$ be the canonical decomposition of $\mathfrak{u}$ with respect to a Hermitian symmetric pair $(U, K)$ and $\mathfrak{a}$ be a maximal abelian subspace of $\mathfrak{p}$. Let $\mathfrak{g}=\mathfrak{k}+\sqrt{-1} \mathfrak{p}$ denote its noncompact dual in the complexification $\mathfrak{u}^{\mathbf{C}}=\mathfrak{g}^{\mathbf{C}}$ and $\mathfrak{g}^{\mathbf{C}}=\mathfrak{k}^{\mathbf{C}}+\mathfrak{p}^{\mathbf{C}}$ their complexification. Let $\mathfrak{c}(\mathfrak{k})$ be the center of $\mathfrak{k}$ and $\mathfrak{h}$ be a maximal abelian subalgebra of $\mathfrak{k}$ and $\mathfrak{u}$ so that $\mathfrak{c}(\mathfrak{k}) \subset \mathfrak{h} \subset \mathfrak{k} \subset \mathfrak{u}$. Let $\Delta$ denote the set of all roots of $\mathfrak{g}^{\mathbf{C}}$ with respect to $\mathfrak{h}^{\mathbf{C}}$ and $\Delta^{+}$denote the set of all positive roots in $\Delta$ relative to a suitable linear order. $\alpha \in \Delta$ is called compact (resp. noncompact) if $\alpha=0$ (resp. $\alpha \neq 0$ ) on $\mathfrak{c}(\mathfrak{k})$. We take the root decompositions with respect to $\mathfrak{h}^{\mathbf{C}}$ :

$$
\mathfrak{u}^{\mathbf{C}}=\mathfrak{h}^{\mathbf{C}}+\sum_{\alpha \in \Delta} \mathfrak{g}^{\alpha}, \quad \mathfrak{k}^{\mathbf{C}}=\mathfrak{h}^{\mathbf{C}}+\sum_{\alpha: \mathrm{cpt}} \mathfrak{g}^{\alpha}, \quad \mathfrak{p}^{\mathbf{C}}=\sum_{\alpha: \text { noncpt }} \mathfrak{g}^{\alpha} .
$$

Set $\Delta_{\mathfrak{c}}:=\{\alpha \in \Delta \mid \alpha \neq 0$ on $\mathfrak{c}(\mathfrak{k})\}$ and $Q_{+}:=\Delta^{+} \cap \Delta_{\mathfrak{c}}$. Then there exist strongly orthogonal roots $\gamma_{1}, \gamma_{2} \in Q_{+}$such that

$$
\mathfrak{a}^{\mathbf{C}}=\sum_{i=1}^{2} \mathbf{C}\left(X_{\gamma_{i}}+X_{-\gamma_{i}}\right)
$$


and

$$
\mathfrak{a}=\sum_{i=1}^{2} \mathbf{R} \sqrt{-1}\left(X_{\gamma_{i}}+X_{-\gamma_{i}}\right) .
$$

Here we use $X_{\alpha} \in \mathfrak{g}^{\alpha}$ such that for each $\alpha \in \Delta$,

$$
\begin{aligned}
& X_{\alpha}-X_{-\alpha}, \sqrt{-1}\left(X_{\alpha}+X_{-\alpha}\right) \in \mathfrak{u} \\
& {\left[X_{\alpha}, X_{-\alpha}\right]=\frac{2}{\alpha\left(H_{\alpha}\right)} H_{\alpha} .}
\end{aligned}
$$

Define an abelian subalgebra $\mathfrak{h}_{\mathfrak{a}}^{\mathbf{C}}$ of $\mathfrak{k}_{0}^{\mathbf{C}}$ by

$$
\mathfrak{h}_{\mathfrak{a}}^{\mathbf{C}}:=\left\{H \in \mathfrak{h}^{\mathbf{C}} \mid \gamma_{i}(H)=\left\langle H_{\gamma_{i}}, H\right\rangle=0(i=1,2)\right\} .
$$

Lemma 3.3. Let $Z \in \mathfrak{c}(\mathfrak{k})$ be a nonzero element. Then $Z \in \mathfrak{m}$ if and only if $Z \perp \mathfrak{h}_{\mathfrak{a}}$, if and only if $Z \in \sum_{i=1}^{2} \mathbf{R} H_{\gamma_{i}}$.

Proof. Suppose that $X_{\alpha} \in \mathfrak{g}^{\alpha}$ with compact $\alpha$ satisfies

$$
\left[X_{\alpha}, X_{\gamma_{i}}+X_{-\gamma_{i}}\right] \in \mathfrak{a}^{\mathbf{C}} \quad(i=1,2) .
$$

Then the condition

$$
0=\left[X_{\alpha}, X_{\gamma_{i}}+X_{-\gamma_{i}}\right]=\left[X_{\alpha}, X_{\gamma_{i}}\right]+\left[X_{\alpha}, X_{-\gamma_{i}}\right] \in \mathfrak{g}^{\alpha+\gamma_{i}}+\mathfrak{g}^{\alpha-\gamma_{i}}
$$

implies that $\left[X_{\alpha}, X_{\gamma_{i}}\right]=0$ and $\left[X_{\alpha}, X_{-\gamma_{i}}\right]=0$. We see that $\alpha+\gamma_{i}$ and $\alpha-\gamma_{i}$ are not roots. Thus we obtain the expression

$$
\mathfrak{k}_{0}^{\mathbf{C}}=\mathfrak{h}_{\mathfrak{a}}^{\mathbf{C}}+\sum_{\alpha} \mathfrak{g}^{\alpha},
$$

where $\alpha \in \Delta$ runs all compact roots such that $\alpha \pm \gamma_{i}$ are not roots for all $i=1,2$. Hence from (3.16) we have

$$
\mathfrak{m}^{\mathbf{C}}=\left(\sum_{i=1}^{2} \mathbf{C} H_{\gamma_{i}}\right)+\sum_{\alpha} \mathfrak{g}^{\alpha},
$$

where $\alpha \in \Delta$ runs all compact roots such that $\alpha+\gamma_{i}$ or $\alpha-\gamma_{i}$ is a root for some $i=1,2$. And we have an orthogonal direct sum decomposition

$$
\mathfrak{h}=\left(\sum_{i=1}^{2} \mathbf{R} H_{\gamma_{i}}\right) \oplus \mathfrak{h}_{\mathfrak{a}} .
$$

From (3.17) and (3.18) we obtain Lemma 3.3.

By using Lemma 3.3, we determine Hermitian symmetric pairs $(U, K)$ of compact type and of rank 2 satisfying the condition (3.10) as follows. We use the table of root systems for complex simple Lie algebras in 8 .

Let $\left\{\alpha_{1}, \cdots, \alpha_{l}\right\}$ be the fundamental root system of $\Delta$. We express the highest root $\tilde{\alpha}$ of $\Delta$ as

$$
\tilde{\alpha}=m_{1} \alpha_{1}+\cdots+m_{l} \alpha_{l} .
$$

Define a basis $\left\{\xi_{1}, \cdots, \xi_{l}\right\}$ of $\mathfrak{h}$ by

$$
\alpha_{i}\left(\xi_{j}\right)=\sqrt{-1}\left\langle H_{\alpha_{i}}, \xi_{j}\right\rangle=\sqrt{-1} \delta_{i j} \quad(i, j=1, \cdots, l) .
$$


The fundamental weight system $\left\{\Lambda_{1}, \cdots, \Lambda_{l}\right\}$ is defined by

$$
2 \frac{\left\langle\Lambda_{j}, \alpha_{i}\right\rangle}{\left\langle\alpha_{i}, \alpha_{i}\right\rangle}=-2 \sqrt{-1} \frac{\alpha_{i}\left(H_{\Lambda_{j}}\right)}{\left\langle\alpha_{i}, \alpha_{i}\right\rangle}=\delta_{i j} \quad(i, j=1, \cdots, l) .
$$

Note that

$$
\xi_{j}=\frac{2 H_{\Lambda_{j}}}{\left\langle\alpha_{j}, \alpha_{j}\right\rangle} \quad(j=1,2, \cdots, l) .
$$

In our case there is a subset $I=\left\{i_{0}\right\}$ of $\{1,2, \cdots, l\}$ with $m_{i_{0}}=1$ such that

$$
\mathfrak{k}^{\mathbf{C}}=\mathfrak{h}^{\mathbf{C}}+\sum_{n_{i_{0}}(\alpha)=0} \mathfrak{g}^{\alpha}, \quad \mathfrak{p}^{\mathbf{C}}=\sum_{n_{i_{0}}(\alpha) \neq 0} \mathfrak{g}^{\alpha},
$$

where we define $n_{i}(\alpha)$ as $\alpha=\sum_{i=1}^{l} n_{i}(\alpha) \alpha_{i} \in \Delta$ (all $n_{i}$ are nonnegative integers or all $n_{i}$ are nonpositive integers). Then we have

$$
\mathfrak{c}(\mathfrak{k})=\mathbf{R} \xi_{i_{0}} \subset \mathbf{C} \xi_{i_{0}}=\mathfrak{c}\left(\mathfrak{k}^{\mathbf{C}}\right)=\mathfrak{c}(\mathfrak{k})^{\mathbf{C}} .
$$

Since $\Delta_{\mathfrak{c}}=\left\{\alpha \in \Delta \mid n_{i_{0}}(\alpha)=1\right\}$, note that $Q_{+}=\Delta^{+} \cap\left\{\alpha \in \Delta \mid n_{i_{0}}=1\right\}$.

$\mathrm{AIII}_{2}$ : The case when $(U, K)=(S U(m+2), S(U(m) \times U(2)))(m \geq 2)$ is described as

$$
\begin{aligned}
& \tilde{\alpha}=\alpha_{1}+\alpha_{2}+\cdots+\alpha_{m+1}=\sqrt{-1}\left(\varepsilon_{1}-\varepsilon_{m+2}\right), H_{\tilde{\alpha}}=e_{1}-e_{m+2}, \\
& i_{0}=2, \alpha_{2}=\sqrt{-1}\left(\varepsilon_{2}-\varepsilon_{3}\right), H_{\alpha_{2}}=e_{2}-e_{3}, \\
& \xi_{2}=\left(e_{1}+e_{2}\right)-\frac{2}{m+2} \sum_{j=1}^{m+2} e_{j} .
\end{aligned}
$$

The strongly orthogonal roots are given by $\gamma_{1}=\alpha_{2}, \gamma_{2}=\tilde{\alpha} \in Q_{+}$. Therefore

$$
\xi_{2} \notin \mathbf{R} H_{\alpha_{2}}+\mathbf{R} H_{\tilde{\alpha}}=\mathbf{R}\left(e_{2}-e_{3}\right)+\mathbf{R}\left(e_{1}-e_{m+2}\right)
$$

if and only if $m \geq 3$. If $m=2$, then

$$
\begin{aligned}
\xi_{2} & =\frac{1}{2}\left(e_{1}+e_{2}\right)-\frac{1}{2}\left(e_{3}+e_{4}\right)=\frac{1}{2}\left(e_{2}-e_{3}\right)+\frac{1}{2}\left(e_{1}-e_{4}\right) \\
& \in \mathbf{R} H_{\alpha_{2}}+\mathbf{R} H_{\tilde{\alpha}}=\mathbf{R}\left(e_{2}-e_{3}\right)+\mathbf{R}\left(e_{1}-e_{4}\right) .
\end{aligned}
$$

Note that $\mathrm{AIII}_{2}(m=2) \cong \mathrm{BDI}_{2}(m=4)$.

$\mathrm{BII}_{2}$ : The case when $(U, K)=(S O(m+2), S O(2) \times S O(m)), m=2 l-1, l \geq$ 2 is described as

$$
\begin{aligned}
& \tilde{\alpha}=\alpha_{1}+2 \alpha_{2}+\cdots+2 \alpha_{l}=\sqrt{-1}\left(\varepsilon_{1}+\varepsilon_{2}\right), H_{\tilde{\alpha}}=e_{1}+e_{2} . \\
& i_{0}=1, \alpha_{1}=\sqrt{-1}\left(\varepsilon_{1}-\varepsilon_{2}\right), H_{\alpha_{1}}=e_{1}-e_{2}, \\
& \xi_{1}=e_{1} .
\end{aligned}
$$

The strongly orthogonal roots are given by $\gamma_{1}=\alpha_{1}, \gamma_{2}=\tilde{\alpha} \in Q_{+}$. Thus

$$
\xi_{1}=e_{1} \in \mathbf{R} H_{\alpha_{1}}+\mathbf{R} H_{\tilde{\alpha}}=\mathbf{R} e_{1}+\mathbf{R} e_{2} .
$$

Hence the condition (3.10) is satisfied in this case. 
$\mathrm{DII}_{2}$ : The case when $(U, K)=(S O(m+2), S O(2) \times S O(m)), m=2 l, l \geq 2$ is described as

$$
\begin{aligned}
& \tilde{\alpha}=\alpha_{1}+2 \alpha_{2}+\cdots+2 \alpha_{l-2}+\alpha_{l-1}+\alpha_{l}=\sqrt{-1}\left(\varepsilon_{1}+\varepsilon_{2}\right), H_{\tilde{\alpha}}=e_{1}+e_{2} . \\
& i_{0}=1, \alpha_{1}=\sqrt{-1}\left(\varepsilon_{1}-\varepsilon_{2}\right), H_{\alpha_{1}}=e_{1}-e_{2}, \\
& \xi_{1}=e_{1} .
\end{aligned}
$$

The strongly orthogonal roots are given by $\gamma_{1}=\alpha_{1}, \gamma_{2}=\tilde{\alpha} \in Q_{+}$.

$$
\xi_{1}=e_{1} \in \mathbf{R} H_{\alpha_{1}}+\mathbf{R} H_{\tilde{\alpha}}=\mathbf{R} e_{1}+\mathbf{R} e_{2} .
$$

Therefore the condition (3.10) is satisfied in this case.

$\mathrm{DIII}_{2}$ : The case when $(U, K)=(S O(10), U(5))$ is described as

$$
\begin{aligned}
& \tilde{\alpha}=\alpha_{1}+2 \alpha_{2}+2 \alpha_{3}+\alpha_{4}+\alpha_{5}=\sqrt{-1}\left(\varepsilon_{1}+\varepsilon_{2}\right), H_{\tilde{\alpha}}=e_{1}+e_{2} . \\
& i_{0}=4, \alpha_{4}=\sqrt{-1}\left(\varepsilon_{4}-\varepsilon_{5}\right), H_{\alpha_{4}}=e_{4}-e_{5}, \\
& \xi_{4}=\frac{1}{2}\left(e_{1}+e_{2}+e_{3}+e_{4}-e_{5}\right) .
\end{aligned}
$$

The strongly orthogonal roots are given by $\gamma_{1}=\alpha_{4}, \gamma_{2}=\tilde{\alpha} \in Q_{+}$. Hence

$$
\xi_{4} \notin \mathbf{R} H_{\alpha_{4}}+\mathbf{R} H_{\tilde{\alpha}} .
$$

Therefore the condition (3.10) is not satisfied in this case.

EIII : The case when $(U, K)=\left(E_{6}, \operatorname{Spin}(10) \cdot T\right)$ is described as

$$
\begin{aligned}
\tilde{\alpha} & =\alpha_{1}+2 \alpha_{2}+2 \alpha_{3}+3 \alpha_{4}+2 \alpha_{5}+\alpha_{6} \\
& =\frac{1}{2} \sqrt{-1}\left(\varepsilon_{1}+\varepsilon_{2}+\varepsilon_{3}+\varepsilon_{4}+\varepsilon_{5}-\varepsilon_{6}-\varepsilon_{7}+\varepsilon_{8}\right), \\
H_{\tilde{\alpha}} & =\frac{1}{2}\left(e_{1}+e_{2}+e_{3}+e_{4}+e_{5}-e_{6}-e_{7}+e_{8}\right) . \\
i_{0} & =1, \alpha_{1}=\frac{1}{2} \sqrt{-1}\left(\varepsilon_{1}+\varepsilon_{8}\right)-\frac{1}{2} \sqrt{-1}\left(\varepsilon_{2}+\varepsilon_{3}+\varepsilon_{4}+\varepsilon_{5}+\varepsilon_{6}+\varepsilon_{7}\right), \\
H_{\alpha_{1}} & =\frac{1}{2}\left(e_{1}+e_{8}\right)-\frac{1}{2}\left(e_{2}+e_{3}+e_{4}+e_{5}+e_{6}+e_{7}\right), \\
\xi_{1} & =\frac{2}{3}\left(e_{8}-e_{7}-e_{6}\right) .
\end{aligned}
$$

The strongly orthogonal roots are given by $\gamma_{1}=\alpha_{1}, \gamma_{2}=\tilde{\alpha} \in Q_{+}$. Hence

$$
\xi_{1} \notin \mathbf{R} H_{\alpha_{1}}+\mathbf{R} H_{\tilde{\alpha}} .
$$

Therefore the condition (3.10) is not satisfied in this case. We complete the proof of Lemma 3.2.

Using (3.13), we set

$$
\begin{aligned}
& 2 H_{1}:=\sqrt{-1}\left(X_{\gamma_{1}}+X_{-\gamma_{1}}\right)-\sqrt{-1}\left(X_{\gamma_{2}}+X_{-\gamma_{2}}\right) \in \mathfrak{a}, \\
& 2 H_{2}:=\sqrt{-1}\left(X_{\gamma_{1}}+X_{-\gamma_{1}}\right)+\sqrt{-1}\left(X_{\gamma_{2}}+X_{-\gamma_{2}}\right) \in \mathfrak{a} .
\end{aligned}
$$

Then the center $\mathfrak{c}(\mathfrak{k})$ of $\mathfrak{k}$ is given as follows : 
Lemma 3.4. Suppose that $\left[\mathrm{BII}_{2}\right](U, K)=(S O(m+2), S O(2) \times S O(m)), m=$ $2 l-1, l \geq 2$ or $\left[\mathrm{DII}_{2}\right]:(U, K)=(S O(m+2), S O(2) \times S O(m)), m=2 l, l \geq 2$. Then we have

$$
0 \neq\left[J H_{2}, H_{2}\right] \in \mathfrak{c}(\mathfrak{k}) .
$$

Proof. Using (3.14) we compute

$$
\begin{aligned}
4\left[J H_{2}, H_{2}\right]= & -\alpha_{1}\left(\xi_{1}\right)\left[X_{\alpha_{1}}-X_{-\alpha_{1}}, X_{\alpha_{1}}+X_{-\alpha_{1}}\right] \\
& -\alpha_{1}\left(\xi_{1}\right)\left[X_{\alpha_{1}}-X_{-\alpha_{1}}, X_{\tilde{\alpha}}+X_{-\tilde{\alpha}}\right] \\
& -\tilde{\alpha}\left(\xi_{1}\right)\left[X_{\tilde{\alpha}}-X_{-\tilde{\alpha}}, X_{\alpha_{1}}+X_{-\alpha_{1}}\right] \\
& -\tilde{\alpha}\left(\xi_{1}\right)\left[X_{\tilde{\alpha}}-X_{-\tilde{\alpha}}, X_{\tilde{\alpha}}+X_{-\tilde{\alpha}}\right] \\
= & -2 \alpha_{1}\left(\xi_{1}\right)\left[X_{\alpha_{1}}, X_{-\alpha_{1}}\right] \\
& -\alpha_{1}\left(\xi_{1}\right)\left(\left[X_{\alpha_{1}}, X_{-\tilde{\alpha}}\right]-\left[X_{-\alpha_{1}}, X_{\tilde{\alpha}}\right]\right) \\
& -\tilde{\alpha}\left(\xi_{1}\right)\left(\left[X_{\tilde{\alpha}}, X_{-\alpha_{1}}\right]-\left[X_{-\tilde{\alpha}}, X_{\alpha_{1}}\right]\right) \\
& -2 \tilde{\alpha}\left(\xi_{1}\right)\left[X_{\tilde{\alpha}}, X_{-\tilde{\alpha}}\right] \\
= & -\alpha_{1}\left(\xi_{1}\right) \cdot 2 \frac{2}{\alpha_{1}\left(H_{\alpha_{1}}\right)} H_{\alpha_{1}}-\tilde{\alpha}\left(\xi_{1}\right) \cdot 2 \frac{2}{\tilde{\alpha}\left(H_{\tilde{\alpha}}\right)} H_{\tilde{\alpha}} \\
= & -2\left(H_{\alpha_{1}}+H_{\tilde{\alpha}}\right)=-4 \xi_{1} \in \mathfrak{c}(\mathfrak{k}) .
\end{aligned}
$$

In this computation we use the following properties of this type (cf. [8]): (i) $\alpha_{1}+\tilde{\alpha} \notin \Delta$. (ii) $\alpha_{1}-\tilde{\alpha} \notin \Delta,-\alpha_{1}+\tilde{\alpha} \notin \Delta$. (iii) $\alpha_{1}\left(H_{\alpha_{1}}\right)=\sqrt{-1}\left\langle H_{\alpha_{1}}, H_{\alpha_{1}}\right\rangle=$ $2 \sqrt{-1}, \tilde{\alpha}\left(H_{\tilde{\alpha}}\right)=\sqrt{-1}\left\langle H_{\tilde{\alpha}}, H_{\tilde{\alpha}}\right\rangle=2 \sqrt{-1}, \alpha_{1}\left(\xi_{1}\right)=\sqrt{-1}\left\langle H_{\alpha_{1}}, \xi_{1}\right\rangle=\sqrt{-1}$, $\tilde{\alpha}\left(\xi_{1}\right)=\sqrt{-1}\left\langle H_{\tilde{\alpha}}, \xi_{1}\right\rangle=\sqrt{-1}$.

\section{Classification of homogeneous Lagrangian Submanifolds in COMPLEX HYPERQUADRICS}

\subsection{Lagrangian orbits and moment maps in complex hyperquadrics.}

Suppose that $K^{\prime} \cdot\left[V_{0}\right] \subset Q_{n}(\mathbf{C})$ is a Lagrangian orbit of a compact connected Lie subgroup $K^{\prime}$ of $S O(n+2)$ through a point $\left[V_{0}\right] \in Q_{n}(\mathbf{C})$. Let $\mu_{K^{\prime}}$ : $Q_{n}(\mathbf{C}) \rightarrow \mathfrak{k}^{\prime}$ denote the moment map for the induced Hamiltonian group action of $K^{\prime}$ on $Q_{n}(\mathbf{C})$. By virtue of Lemma 2.3 there is a unit vector $v \in V_{0}$ such that the orbit $K^{\prime} \cdot v \subset S^{n+1}(1)$ through $v$ under the group $K^{\prime}$ is a homogeneous isoparametric hypersurface embedded in $S^{n+1}(1)$. Thus by Hsiang-Lawson's theorem ([16]) there is a Riemannian symmetric pair $(U, K)$ of compact type and of rank 2 with compact connected $K$ such that $\mathfrak{p}=\mathbf{R}^{n+2}, K^{\prime} \subset \operatorname{Ad}_{\mathfrak{p}}(K)$, and $K^{\prime} \cdot v=\operatorname{Ad}_{\mathfrak{p}}(K) v$, where $\mathfrak{u}=\mathfrak{k}+\mathfrak{p}$ denotes the canonical decomposition of the symmetric Lie algebra $(\mathfrak{u}, \mathfrak{k})$ of $(U, K)$. Then we can show that $K^{\prime} \cdot\left[V_{0}\right]=$ $K \cdot\left[V_{0}\right]$ as follows:

According to the classification due to T. Asoh ([4]), if $K^{\prime} \neq \operatorname{Ad}_{\mathfrak{p}}(K)$, then one of the following cases happens :

(i) $K^{\prime}$ is semisimple, i.e., $\mathfrak{c}\left(\mathfrak{k}^{\prime}\right)=\{0\}$.

(ii) $K^{\prime}$ is not semisimple and $K^{\prime}=K_{s s}^{\prime} \times C\left(K^{\prime}\right)$ where $K_{s s}^{\prime}$ is semisimple such that $K_{s s}^{\prime}$ acts on $S^{n+1}(1)$ with cohomogeneity 1 . 
(iii) $K^{\prime}$ is not semisimple and $K^{\prime}=K_{s s}^{\prime} \times C\left(K^{\prime}\right)$ where $K_{s s}^{\prime}$ is semisimple such that $K_{s s}^{\prime}$ does NOT act on $S^{n+1}(1)$ with cohomogeneity 1 . In this case

(a) $K^{\prime}=S O(2) \times G_{2}, n=12,(U, K)=(S O(9), S O(2) \times S O(7))$.

(b) $K^{\prime}=S O(2) \times S p i n(7), n=14,(U, K)=(S O(10), S O(2) \times S O(8))$.

In case (i), since $\mathfrak{c}\left(\mathfrak{k}^{\prime}\right)=\{0\}, K^{\prime}$ has only one Lagrangian orbit $K^{\prime} \cdot\left[V_{0}\right]=$ $\mu_{K^{\prime}}^{-1}(0)=\mathcal{G}\left(K^{\prime} \cdot v\right)=\mathcal{G}(K \cdot v)=\mu_{K}^{-1}(0)=K \cdot\left[V_{0}\right]$ on $Q_{n}(\mathbf{C})$.

In case (ii), since $K_{s s}^{\prime} \cdot\left[V_{0}\right] \subset K^{\prime} \cdot\left[V_{0}\right]=\left(K_{s s}^{\prime} \times C\left(K^{\prime}\right)\right) \cdot\left[V_{0}\right]$, we see that $K_{s s}^{\prime} \cdot\left[V_{0}\right] \subset Q_{n}(\mathbf{C})$ is an isotropic orbit. Thus $K_{s s}^{\prime} \cdot\left[V_{0}\right] \subset \mu_{K_{s s}^{\prime}}^{-1}(0)$. On the other hand $\mu_{K_{s s}^{\prime}}^{-1}(0)=\mathcal{G}\left(K_{s s}^{\prime} \cdot v\right)=\mathcal{G}\left(K^{\prime} \cdot v\right)=\mathcal{G}(K \cdot v)=K_{s s}^{\prime} \cdot \mathcal{G}(v)$ is a Lagrangian orbit. Hence $K_{s s}^{\prime} \cdot\left[V_{0}\right]=K_{s s}^{\prime} \cdot \mathcal{G}(v)=\mathcal{G}\left(K_{s s}^{\prime} \cdot v\right)=\mathcal{G}\left(K^{\prime} \cdot v\right)=\mathcal{G}(K \cdot v)=$ $K^{\prime} \cdot\left[V_{0}\right]=K \cdot\left[V_{0}\right] \subset Q_{n}(\mathbf{C})$.

In case (iii), we may assume one of the following :

(a) $K^{\prime}=S O(2) \times G_{2}, n=12,(U, K)=(S O(9), S O(2) \times S O(7))$ and $K_{0}=\mathbf{Z}_{2} \times S O(5), K_{0}^{\prime}=K^{\prime} \cap K_{0}=\mathbf{Z}_{2} \times S U(2)$.

(b) $K^{\prime}=S O(2) \times \operatorname{Spin}(7), n=14,(U, K)=(S O(10), S O(2) \times S O(8))$ and $K_{0}=\mathbf{Z}_{2} \times S O(6), K_{0}^{\prime}=K^{\prime} \cap K_{0}=\mathbf{Z}_{2} \times S U(3)$.

Since $K^{\prime} \cdot v=K \cdot v$, we can assume that $v \in \mathfrak{a}$. We take the orthogonal direct sum $\mathfrak{k}=\mathfrak{k}^{\prime}+\mathfrak{k}^{\prime \perp}$. And we have $\mathfrak{k}_{0}=\{\xi \in \mathfrak{k} \mid[\xi, v]=0\}$. Set $\mathfrak{k}_{0}^{\prime}=\mathfrak{k}_{0} \cap \mathfrak{k}^{\prime}$ and we take the orthogonal direct sum $\mathfrak{k}_{0}=\mathfrak{k}_{0}^{\prime}+\mathfrak{k}_{0}^{\prime \perp}$. Then we obtain $\mathfrak{k}^{\perp}=\mathfrak{k}_{0}^{\prime \perp}$. Let $\{v, j v\}$ be an orthonormal basis of $V_{0}$ compatible with its orientation. Since $\mu_{K^{\prime}}\left(\left[V_{0}\right]\right)=-[v, j v]_{\mathfrak{k}^{\prime}} \in \mathfrak{c}\left(\mathfrak{k}^{\prime}\right)$, we can express $[v, j v]$ as

$$
[v, j v]=\eta+\zeta
$$

where $\eta \in \mathfrak{c}\left(\mathfrak{k}^{\prime}\right)$ and $\zeta \in \mathfrak{k}^{\perp \perp}$. Since $v \in \mathfrak{a}$ and $\mathfrak{k}^{\perp}=\mathfrak{k}_{0}^{\prime \perp} \subset \mathfrak{k}_{0}$, we have

$$
[v,[v, j v]]=[v, \eta]
$$

and so

$$
\begin{aligned}
\langle[v,[v, j v]], j v\rangle_{\mathfrak{u}} & =\langle[v, \eta], j v\rangle_{\mathfrak{u}} \\
-\langle[v, j v],[v, j v]\rangle_{\mathfrak{u}} & =-\langle\eta,[v, j v]\rangle_{\mathfrak{u}} \\
-\langle\eta, \eta\rangle_{\mathfrak{u}}-\langle\zeta, \zeta\rangle_{\mathfrak{u}} & =-\langle\eta, \eta\rangle_{\mathfrak{u}} \\
\langle\zeta, \zeta\rangle_{\mathfrak{u}} & =0 .
\end{aligned}
$$

Thus $\zeta=0$ and $\mu_{K}\left(\left[V_{0}\right]\right)=-[v, j v]=\eta \in \mathfrak{c}\left(\mathfrak{k}^{\prime}\right)=\mathfrak{c}(\mathfrak{k})$, that is, $K \cdot\left[V_{0}\right] \subset Q_{n}(\mathbf{C})$ is an isotropic orbit. Hence $K^{\prime} \cdot\left[V_{0}\right]=K \cdot\left[V_{0}\right] \subset Q_{n}(\mathbf{C})$ is a Lagrangian orbit.

Therefore we may assume that $K=K^{\prime}$ and thus $\mathfrak{k}=\mathfrak{k}^{\prime}$. We choose an orthonormal basis $\left\{v_{1}, v_{2}\right\}$ of $V_{0}$ compatible with the orientation of $\left[V_{0}\right]$. By the adjoint action of $K$ on $\mathfrak{p}$ we can assume that $v_{1} \in \mathfrak{a} \cap S^{n+1}(1)$. Following the decomposition (3.5), we decompose $v_{2}$ as

$$
v_{2}=v_{2,0}+\sum_{\gamma \in \Sigma^{+}(U, K)} v_{2, \gamma}
$$


where $v_{2,0} \in \mathfrak{a}$ and $v_{2, \gamma} \in \mathfrak{p}_{\gamma}$. Then it follows from $\left[v_{1}, v_{2, \gamma}\right] \in \mathfrak{k}_{\gamma}$ that

$$
\begin{aligned}
\mathfrak{c}(\mathfrak{k}) \ni \mu\left(\left[V_{0}\right]\right) & =-\left[v_{1}, v_{2}\right] \\
& =-\left[v_{1}, v_{2,0}+\sum_{\gamma \in \Sigma^{+}(U, K)} v_{2, \gamma}\right] \\
& =-\sum_{\gamma \in \Sigma^{+}(U, K)}\left[v_{1}, v_{2, \gamma}\right] \in \mathfrak{m} .
\end{aligned}
$$

Set $\xi_{0}:=\mu\left(\left[V_{0}\right]\right) \in \mathfrak{c}(\mathfrak{k}) \cap \mathfrak{m}$.

In the case when $\mathfrak{c}(\mathfrak{k}) \cap \mathfrak{m}=\{0\}$, we have $\mu\left(\left[V_{0}\right]\right)=0$ and thus $K \cdot\left[V_{0}\right] \subset$ $\mu^{-1}(0)$. Hence since $\mu^{-1}(0)$ is connected by Proposition 1.3 (1), we obtain $K \cdot\left[V_{0}\right]=\mu^{-1}(0)=K \cdot[\mathfrak{a}]$.

Suppose that $\mathfrak{c}(\mathfrak{k}) \cap \mathfrak{m} \neq\{0\}$, or equivalently $\{0\} \neq \mathfrak{c}(\mathfrak{k}) \subset \mathfrak{m}$. By Lemma 3.2. $(U, K)$ must be one of the following :

(a) $\left(S^{1} \times S O(3), S O(2)\right)$,

(b) $(S O(3) \times S O(3), S O(2) \times S O(2))$,

(c) $(S O(3) \times S O(n+1), S O(2) \times S O(n))(n \geq 3)$,

(d) $(S O(m+2), S O(2) \times S O(m))(n=2 m-2, m \geq 3)$.

Lemma 4.1. Set

$$
s:=\operatorname{Max}\left\{\|\mu([V])\|^{2} \mid[V] \in \widetilde{\operatorname{Gr}}_{2}(\mathfrak{p}) \text { with } \mu([V]) \in \mathfrak{c}(\mathfrak{k})\right\}
$$

(a) If $(U, K)=\left(S^{1} \times S O(3), S O(2)\right)$, then the value $s$ is equal to the maximum of sectional curvatures of $\left(U / K, g_{U}\right)=S^{1} \times(S O(3) / S O(2))$. Moreover $\|\mu([V])\|^{2}$ attains the maximum if and only if $[V]$ is an oriented 2-dimensional subspace of $\mathfrak{p}$ tangent to $S O(3) / S O(2)$.

(b) If $(U, K)=(S O(3) \times S O(3), S O(2) \times S O(2))$, then the value $s$ is equal to the maximum of sectional curvatures of $\left(U / K, g_{U}\right)=(S O(3) / S O(2)) \times$ $(S O(3) / S O(2))$. Moreover $\|\mu([V])\|^{2}$ attains the maximum if and only if $[V]$ is an oriented 2 -dimensional subspace of $\mathfrak{p}$ tangent to $S O(3) / S O(2)$ with larger curvature.

(c) If $(U, K)=(S O(3) \times S O(n+1), S O(2) \times S O(n))(n \geq 3)$, then the value $s$ is equal to the sectional curvature of the first factor $S O(3) / S O(2)$ of $\left(U / K, g_{U}\right)=(S O(3) / S O(2)) \times(S O(n+1) / S O(n))$. Moreover $\|\mu([V])\|^{2}$ is equal to the sectional curvature of the first factor $S O(3) / S O(2)$ if and only if $[V]$ is an oriented 2-dimensional subspace of $\mathfrak{p}$ tangent to $S O(3) / S O(2)$.

(d) If $(U, K)=(S O(m+2), S O(2) \times S O(m))(n=2 m-2, m \geq 3)$, then the value $s$ is equal to the minimum of holomorphic sectional curvatures of $\left(U / K, g_{U}, J\right)=S O(m+2) /(S O(2) \times S O(m))=Q_{m}(\mathbf{C})$. Moreover $\|\mu([V])\|^{2}$ is equal to the minimum of holomorphic sectional curvatures of $\left(U / K, g_{U}\right)$ if and only if $[V]$ is an oriented 2-dimensional vector subspace of $\mathfrak{p}$ spanned by a vector $H_{\gamma}$ dual to a short root $\gamma \in \Sigma(U, K)$ and $J H_{\gamma}$, up to the adjoint action of $K$ on $\mathfrak{p}$. 
Proof. (a) Let $(U, K)=\left(S^{1} \times S O(3), S O(2)\right)$. Then

$$
\begin{aligned}
& \mathfrak{u}=\sqrt{-1} \mathbf{R} \oplus \mathfrak{s o}(3), \quad \mathfrak{k}=\mathfrak{s o}(2)=\mathfrak{c}(\mathfrak{k})=\left\{\left(\begin{array}{ccc}
0 & 0 & 0 \\
0 & 0 & c \\
0 & -c & 0
\end{array}\right) \mid c \in \mathbf{R}\right\} \\
& \mathfrak{p}=\sqrt{-1} \mathbf{R} \oplus\left\{\left(\begin{array}{ccc}
0 & x & y \\
-x & 0 & 0 \\
-y & 0 & 0
\end{array}\right) \mid x, y \in \mathbf{R}\right\}, \\
& \mathfrak{a}=\sqrt{-1} \mathbf{R} \oplus\left\{\left(\begin{array}{ccc}
0 & x & 0 \\
-x & 0 & 0 \\
0 & 0 & 0
\end{array}\right) \mid x \in \mathbf{R}\right\} .
\end{aligned}
$$

Set

$$
H_{1}:=(\sqrt{-1}, 0) \in \sqrt{-1} \mathbf{R} \oplus\{0\} \subset \mathfrak{a}, \quad H_{2}:=\left(\begin{array}{ccc}
0 & 1 & 0 \\
-1 & 0 & 0 \\
0 & 0 & 0
\end{array}\right) \in \mathfrak{a} .
$$

In this case we define $J:=\operatorname{ad}_{\mathfrak{p}} Z$ by

$$
Z=\left(\begin{array}{ccc}
0 & 0 & 0 \\
0 & 0 & 1 \\
0 & -1 & 0
\end{array}\right)
$$

Then we obtain

$$
\left[\cos \theta H_{1}+\sin \theta J H_{2}, H_{2}\right]=\sin \theta\left[J H_{2}, H_{2}\right]=(\sin \theta) Z
$$

and

$$
\left\|\left[\cos \theta H_{1}+\sin \theta J H_{2}, H_{2}\right]\right\|^{2}=|\sin \theta|^{2}\|Z\|^{2} .
$$

This implies the statement (a).

(b) Let $(U, K)=(S O(3) \times S O(3), S O(2) \times S O(2))$. Then

$$
\begin{aligned}
& \mathfrak{u}=\mathfrak{s} \mathfrak{o}(3) \oplus \mathfrak{s o}(3), \quad \mathfrak{k}=\mathfrak{s o}(2) \oplus \mathfrak{s o}(2)=\mathfrak{c}(\mathfrak{k}), \\
& \mathfrak{p}=\left\{\left(\left(\begin{array}{ccc}
0 & x & y \\
-x & 0 & 0 \\
-y & 0 & 0
\end{array}\right),\left(\begin{array}{ccc}
0 & x^{\prime} & y^{\prime} \\
-x^{\prime} & 0 & 0 \\
-y^{\prime} & 0 & 0
\end{array}\right)\right) \mid x, y, x^{\prime}, y^{\prime} \in \mathbf{R}\right\}, \\
& \mathfrak{a}=\left\{\left(\left(\begin{array}{ccc}
0 & x & 0 \\
-x & 0 & 0 \\
0 & 0 & 0
\end{array}\right),\left(\begin{array}{ccc}
0 & x^{\prime} & 0 \\
-x^{\prime} & 0 & 0 \\
0 & 0 & 0
\end{array}\right)\right) \mid x, x^{\prime} \in \mathbf{R}\right\} .
\end{aligned}
$$

Let

$$
v_{1}=\left(\left(\begin{array}{ccc}
0 & x_{1} & 0 \\
-x_{1} & 0 & 0 \\
0 & 0 & 0
\end{array}\right),\left(\begin{array}{ccc}
0 & x_{1}^{\prime} & 0 \\
-x_{1}^{\prime} & 0 & 0 \\
0 & 0 & 0
\end{array}\right)\right) \in \mathfrak{a}
$$

with $\left(x_{1}\right)^{2}+\left(x_{1}^{\prime}\right)^{2}=1$. We may assume that $x_{1} \geq 0$ and $x_{1}^{\prime} \geq 0$. Let

$$
v_{2}=\left(\left(\begin{array}{ccc}
0 & x_{2} & y_{2} \\
-x_{2} & 0 & 0 \\
-y_{2} & 0 & 0
\end{array}\right),\left(\begin{array}{ccc}
0 & x_{2}^{\prime} & y_{2}^{\prime} \\
-x_{2}^{\prime} & 0 & 0 \\
-y_{2}^{\prime} & 0 & 0
\end{array}\right)\right) \in \mathfrak{p}
$$


with $x_{2}^{2}+y_{2}^{2}+{x_{2}^{\prime}}^{2}+y_{2}^{\prime 2}=1$. Then

$$
\mathfrak{k}=\mathfrak{c}(\mathfrak{k}) \ni\left[v_{1}, v_{2}\right]=\left(x_{1} y_{2}\left(\begin{array}{ccc}
0 & 0 & 0 \\
0 & 0 & -1 \\
0 & 1 & 0
\end{array}\right), x_{1}^{\prime} y_{2}^{\prime}\left(\begin{array}{ccc}
0 & 0 & 0 \\
0 & 0 & -1 \\
0 & 1 & 0
\end{array}\right)\right) \in \mathfrak{c}(\mathfrak{k})=\mathfrak{k}
$$

and thus

$$
\begin{aligned}
\left\|\left[v_{1}, v_{2}\right]\right\|^{2} & =\left(x_{1} y_{2}\right)^{2}+\left(x_{1}^{\prime} y_{2}^{\prime}\right)^{2} \\
& =\left(x_{1}\right)^{2}+\left(x_{1}^{\prime}\right)^{2} \leq 1 .
\end{aligned}
$$

Here $\left\|\left[v_{1}, v_{2}\right]\right\|^{2}=1$ if and only if

$$
\left(y_{2}\right)^{2}=1 \text { or }\left(y_{2}^{\prime}\right)^{2}=1 \text {, i.e., } y_{2}= \pm 1 \text { or } y_{2}^{\prime}= \pm 1,
$$

if and only if

$$
v_{1}=\left(\left(\begin{array}{ccc}
0 & 1 & 0 \\
-1 & 0 & 0 \\
0 & 0 & 0
\end{array}\right), 0\right), \quad v_{2}=\left(\left(\begin{array}{ccc}
0 & 0 & \pm 1 \\
0 & 0 & 0 \\
\mp 1 & 0 & 0
\end{array}\right), 0\right)
$$

or

$$
v_{1}=\left(0,\left(\begin{array}{ccc}
0 & 1 & 0 \\
-1 & 0 & 0 \\
0 & 0 & 0
\end{array}\right)\right), \quad v_{2}=\left(0,\left(\begin{array}{ccc}
0 & 0 & \pm 1 \\
0 & 0 & 0 \\
\mp 1 & 0 & 0
\end{array}\right)\right) .
$$

This implies the statement (b).

(c) Let $(U, K)=(S O(3) \times S O(n+1), S O(2) \times S O(n))(n \geq 3)$.

$$
\begin{aligned}
& \mathfrak{u}=\mathfrak{s o}(3) \oplus \mathfrak{s o}(n+1), \mathfrak{k}=\mathfrak{s o}(2) \oplus \mathfrak{s o}(n), \mathfrak{c}(\mathfrak{k})=\mathfrak{s o}(2) \oplus\{0\}, \\
& \mathfrak{p}=\left\{\left(\left(\begin{array}{ccc}
0 & x & y \\
-x & 0 & 0 \\
-y & 0 & 0
\end{array}\right),\left(\begin{array}{cc}
0 & X \\
-{ }^{t} X & 0
\end{array}\right)\right) \mid x, y \in \mathbf{R}, X \in M(1, n ; \mathbf{R})\right\}, \\
& \mathfrak{a}=\left\{\left(\left(\begin{array}{ccc}
0 & x & 0 \\
-x & 0 & 0 \\
0 & 0 & 0
\end{array}\right),\left(\begin{array}{ccccc}
0 & y & 0 & \cdots & 0 \\
-y & 0 & 0 & \cdots & 0 \\
0 & 0 & 0 & \cdots & 0 \\
\vdots & \vdots & \vdots & \cdots & \vdots \\
0 & 0 & 0 & \cdots & 0
\end{array}\right) \mid x, y \in \mathbf{R}\right\} .\right.
\end{aligned}
$$

In this case we define $J:=\operatorname{ad}_{\mathfrak{p}} Z$ by

$$
Z=\left(\left(\begin{array}{ccc}
0 & 0 & 0 \\
0 & 0 & -1 \\
0 & 1 & 0
\end{array}\right), 0\right)
$$

If we set

$$
\left.H_{1}=\left(\begin{array}{ccccc}
0 & 1 & 0 & \cdots & 0 \\
-1 & 0 & 0 & \cdots & 0 \\
0 & 0 & 0 & \cdots & 0 \\
\vdots & \vdots & \vdots & \cdots & \vdots \\
0 & 0 & 0 & \cdots & 0
\end{array}\right)\right), \quad H_{2}=\left(\left(\begin{array}{ccc}
0 & 1 & 0 \\
-1 & 0 & 0 \\
0 & 0 & 0
\end{array}\right), 0\right)
$$


then

Let

$$
J H_{2}=\left[Z, H_{2}\right]=\left(\left(\begin{array}{ccc}
0 & 0 & 1 \\
0 & 0 & 0 \\
-1 & 0 & 0
\end{array}\right), 0\right)
$$

$$
\mathfrak{a} \ni v_{1}=\left(\left(\begin{array}{ccc}
0 & x & 0 \\
-x & 0 & 0 \\
0 & 0 & 0
\end{array}\right),\left(\begin{array}{ccccc}
0 & y & 0 & \cdots & 0 \\
-y & 0 & 0 & \cdots & 0 \\
0 & 0 & 0 & \cdots & 0 \\
\vdots & \vdots & \vdots & \cdots & \vdots \\
0 & 0 & 0 & \cdots & 0
\end{array}\right)\right)
$$

with $x^{2}+y^{2}=1$. We may assume that $x \geq 0$. Let

$$
\mathfrak{p} \ni v_{2}=\left(\left(\begin{array}{ccc}
0 & x^{\prime} & y^{\prime} \\
-x^{\prime} & 0 & 0 \\
-y^{\prime} & 0 & 0
\end{array}\right),\left(\begin{array}{cc}
0 & X \\
-{ }^{t} X & 0
\end{array}\right)\right)
$$

with $x^{\prime 2}+y^{\prime 2}+X^{t} X=1$. Then

$$
\mathfrak{c}(\mathfrak{k}) \ni\left[v_{1}, v_{2}\right]=\left(x y^{\prime}\left(\begin{array}{ccc}
0 & 0 & 0 \\
0 & 0 & -1 \\
0 & 1 & 0
\end{array}\right), y\left(\begin{array}{ccccc}
0 & 0 & 0 & \cdots & 0 \\
0 & 0 & -X_{2} & \cdots & -X_{n} \\
0 & X_{2} & 0 & \cdots & 0 \\
\vdots & \vdots & \vdots & \cdots & \vdots \\
0 & X_{n} & 0 & \cdots & 0
\end{array}\right)\right)
$$

if and only if $y X_{2}=\cdots=y X_{n}=0$. Suppose that $\left[v_{1}, v_{2}\right] \in \mathfrak{c}(\mathfrak{k})$. Then

$$
\left\|\left[v_{1}, v_{2}\right]\right\|^{2}=\left(x y^{\prime}\right)^{2}+\sum_{i=2}^{n}\left(y X_{i}\right)^{2}=\left(x y^{\prime}\right)^{2} \leq 1
$$

Here $\left\|\left[v_{1}, v_{2}\right]\right\|^{2}=1$ if and only if

$$
x^{2}=1 \text { and }\left(y^{\prime}\right)^{2}=1 \text {, i.e., } x=1 \text { and } y^{\prime}= \pm 1,
$$

if and only if

$$
v_{1}=\left(\left(\begin{array}{ccc}
0 & 1 & 0 \\
-1 & 0 & 0 \\
0 & 0 & 0
\end{array}\right), 0\right)=H_{2}, \quad v_{2}=\left(\left(\begin{array}{ccc}
0 & 0 & \pm 1 \\
0 & 0 & 0 \\
\mp 1 & 0 & 0
\end{array}\right), 0\right)= \pm J H_{2} .
$$

This implies the statement (c).

(d) Let $(U, K)=(S O(m+2), S O(2) \times S O(m))(n=2 m-2, m \geq 3)$. Suppose that $\mu([V]) \in \mathfrak{c}(\mathfrak{k})$. We can choose an orthonormal basis $\left\{v_{1}, v_{2}\right\}$ of $V$ compatible with the orientation of $[V]$ such that $v_{1} \in \mathfrak{a} \cap S^{n+1}(1)$ and $(-\sqrt{-1}) \gamma\left(v_{1}\right) \geq 0$ for each $\gamma \in \Sigma^{+}(U, K)$. We express $v_{2}$ as

$$
v_{2}=v_{2,0}+\sum_{\gamma \in \Sigma^{+}(U, K)} v_{2, \gamma}
$$

where $v_{2,0} \in \mathfrak{a}$ and $v_{2, \gamma} \in \mathfrak{p}_{\gamma}$. In this case

$$
\begin{aligned}
& \Sigma^{+}(U, K)=\left\{\gamma_{1}=\sqrt{-1}\left(\varepsilon_{1}-\varepsilon_{2}\right), \gamma_{2}=\sqrt{-1} \varepsilon_{2}, \gamma_{1}+\gamma_{2}=\sqrt{-1} \varepsilon_{1},\right. \\
& \left.\tilde{\gamma}=\gamma_{1}+2 \gamma_{2}=\sqrt{-1}\left(\varepsilon_{1}+\varepsilon_{2}\right)\right\}
\end{aligned}
$$


and set

$$
\left\{H_{\gamma_{1}}=e_{1}-e_{2}, H_{\gamma_{2}}=e_{2}, H_{\gamma_{1}+\gamma_{2}}=e_{1}, H_{\tilde{\gamma}}=H_{\gamma_{1}+2 \gamma_{2}}=e_{1}+e_{2}\right\} \subset \mathfrak{a} .
$$

Then $\left\{H_{1}:=e_{2}, H_{2}:=e_{1}\right\}$ is an orthonormal basis of $\mathfrak{a}$, which defines an orientation of $\mathfrak{a}$, and we have the root decomposition of $\mathfrak{a}^{\perp}$ with respect to $\Sigma^{+}(U, K)$ as

$$
\mathfrak{a}^{\perp}=\sum_{\gamma \in \Sigma^{+}(U, K)} \mathfrak{p}_{\gamma}=\mathfrak{p}_{\gamma_{1}}+\mathfrak{p}_{\gamma_{2}}+\mathfrak{p}_{\gamma_{1}+\gamma_{2}}+\mathfrak{p}_{\gamma_{1}+2 \gamma_{2}},
$$

where $\operatorname{dim} \mathfrak{p}_{\gamma_{1}}=1, \operatorname{dim} \mathfrak{p}_{\gamma_{2}}=m-2, \operatorname{dim} \mathfrak{p}_{\gamma_{1}+\gamma_{2}}=m-2, \operatorname{dim} \mathfrak{p}_{\gamma_{1}+2 \gamma_{2}}=1$. Using the orthonormal basis (3.7), (3.8), we express each $v_{2, \gamma}$ as

$$
v_{2, \gamma}=\sum_{i=1}^{m(\gamma)} c_{\gamma, i} Y_{\gamma, i}
$$

where $c_{\gamma, i} \in \mathbf{R}$. Then we have

$$
v_{2}=v_{2,0}+c_{\gamma_{1}, 1} Y_{\gamma_{1}, 1}+c_{\tilde{\gamma}, 1} Y_{\tilde{\gamma}, 1}+\sum_{i=1}^{m-2} c_{\gamma_{2}, i} Y_{\gamma_{2}, i}+\sum_{i=1}^{m-2} c_{\gamma_{1}+\gamma_{2}, i} Y_{\gamma_{1}+\gamma_{2}, i}
$$

and

$$
\left\|v_{2}\right\|^{2}=\left\|v_{2,0}\right\|^{2}+\left|c_{\gamma_{1}, 1}\right|^{2}+\left|c_{\tilde{\gamma}, 1}\right|^{2}+\sum_{i=1}^{m-2}\left|c_{\gamma_{2}, i}\right|^{2}+\sum_{i=1}^{m-2}\left|c_{\gamma_{1}+\gamma_{2}, i}\right|^{2}=1 .
$$

Thus

$$
\begin{aligned}
& {\left[v_{1}, v_{2}\right] \in \mathfrak{c}(\mathfrak{k})=\mathbf{R}\left(X_{\gamma_{1}}+X_{\tilde{\gamma}}\right) } \\
= & c_{\gamma_{1}, 1}\left[v_{1}, Y_{\gamma_{1}, 1}\right]+c_{\tilde{\gamma}, 1}\left[v_{1}, Y_{\tilde{\gamma}, 1}\right]+\sum_{i=1}^{m-2} c_{\gamma_{2}, i}\left[v_{1}, Y_{\gamma_{2}, i}\right]+\sum_{i=1}^{m-2} c_{\gamma_{1}+\gamma_{2}, i}\left[v_{1}, Y_{\gamma_{1}+\gamma_{2}, i}\right] \\
= & c_{\gamma_{1}, 1}\left(-\sqrt{-1} \gamma_{1}\left(v_{1}\right) X_{\gamma_{1}}\right)+c_{\tilde{\gamma}, 1}\left(-\sqrt{-1} \tilde{\gamma}\left(v_{1}\right) X_{\tilde{\gamma}}\right) \\
= & -c_{\gamma_{1}, 1}\left(\sqrt{-1} \gamma_{1}\left(v_{1}\right)\right) X_{\gamma_{1}}-c_{\tilde{\gamma}, 1}\left(\sqrt{-1} \tilde{\gamma}\left(v_{1}\right)\right) X_{\tilde{\gamma}} \\
= & -c_{\gamma_{1}, 1}\left(v_{1,1}-v_{1,2}\right) X_{\gamma_{1}}-c_{\tilde{\gamma}, 1}\left(v_{1,1}+v_{1,2}\right) X_{\tilde{\gamma}},
\end{aligned}
$$

where $v_{1}=v_{1,1} e_{1}+v_{1,2} e_{2} \in \mathfrak{a}$. Note that $v_{1,1}^{2}+v_{1,2}^{2}=1, v_{1,1} \geq 0, v_{1,2} \geq 0$, $v_{1,1}-v_{1,2} \geq 0$, and $v_{1,1}+v_{1,2} \geq 0$. Hence we have

$$
c_{\gamma_{1}}\left(v_{1,1}-v_{1,2}\right)=c_{\tilde{\gamma}}\left(v_{1,1}+v_{1,2}\right)
$$

and

$$
\left|c_{\gamma_{1}, 1}\right|\left(v_{1,1}-v_{1,2}\right)=\left|c_{\tilde{\gamma}, 1}\right|\left(v_{1,1}+v_{1,2}\right) .
$$

It implies that $\left|c_{\gamma_{1}, 1}\right| \geq\left|c_{\tilde{\gamma}, 1}\right|$. Therefore we obtain

$$
\begin{aligned}
\left\|\left[v_{1}, v_{2}\right]\right\|^{2}= & \left|c_{\gamma_{1}}\right|^{2}\left|v_{1,1}-v_{1,2}\right|^{2}+\left|c_{\tilde{\gamma}}\right|^{2}\left|v_{1,1}+v_{1,2}\right|^{2} \\
= & \left|c_{\gamma_{1}, 1}\right|^{2}\left(v_{1,1}^{2}-2 v_{1,1} v_{1,2}+v_{1,2}^{2}\right) \\
& +\left|c_{\tilde{\gamma}, 1}\right|^{2}\left(v_{1,1}^{2}+2 v_{1,1} v_{1,2}+v_{1,2}^{2}\right) \\
= & \left|c_{\gamma_{1}, 1}\right|^{2}\left(1-2 v_{1,1} v_{1,2}\right)+\left|c_{\tilde{\gamma}, 1}\right|^{2}\left(1+2 v_{1,1} v_{1,2}\right) \\
= & \left|c_{\gamma_{1}, 1}\right|^{2}+\left|c_{\tilde{\gamma}, 1}\right|^{2}+2 v_{1,1} v_{1,2}\left(\left|c_{\tilde{\gamma}, 1}\right|^{2}-\left|c_{\gamma_{1}, 1}\right|^{2}\right) \\
\leq & 1+2 v_{1,1} v_{1,2}\left(\left|c_{\tilde{\gamma}, 1}\right|^{2}-\left|c_{\gamma_{1}, 1}\right|^{2}\right) \leq 1 .
\end{aligned}
$$


Here $\left\|\left[v_{1}, v_{2}\right]\right\|^{2}=1$ if and only if $v_{1}=e_{1}=H_{2}$ and $v_{2}= \pm \frac{1}{\sqrt{2}}\left(Y_{\gamma_{1}, 1}+Y_{\tilde{\gamma}, 1}\right)=$ $\pm \mathrm{JH}_{2}$. This implies the statement (d).

4.2. Two one-parameter families of Lagrangian orbits in complex hyperquadrics. In the cases of $(U, K)=\left(S^{1} \times S O(3), S O(2)\right)$ and $(U, K)=$ $(S O(3) \times S O(3), S O(2) \times S O(2))$, the induced action of $K=S O(2)$ on $Q_{1}(\mathbf{C})$ is just the standard $S^{1}$-action on $S^{2}$ via the identification $Q_{1}(\mathbf{C}) \cong S^{2}$ and the induced action of $K=S O(2) \times S O(2)$ on $Q_{2}(\mathbf{C})$ is the product standard $S^{1} \times S^{1}$-action on $S^{2} \times S^{2}$ via the identification $Q_{2}(\mathbf{C}) \cong S^{2} \times S^{2}$, respectively. In the first case every $K$-orbit except for two fixed points (0dimensional isotropic orbits) is a Lagrangian orbit. In the second case every $K$-orbit except for four fixed points (0-dimensional isotropic orbits) and for four one-parameter families of 1-dimensional isotropic orbits is a Lagrangian orbit.

Suppose that $(U, K)$ is $(S O(3) \times S O(n+1), S O(2) \times S O(n))(n \geq 3)$ or $(S O(m+2), S O(2) \times S O(m))(n=2 m-2, m \geq 3)$.

Let $\left\{H_{1}, H_{2}\right\}$ be an orthonormal basis of $\mathfrak{a}$, which defines an orientation of $\mathfrak{a}$, described in (3.20) or the proof of Lemma 4.1 (c), (d). For each $\lambda=e^{\sqrt{-1} \theta} \in S^{1}$, we define a 2 -dimensional vector subspace $W_{\lambda}$ of $\mathfrak{p}$ by

$$
W_{\lambda}:=\mathbf{R}\left(\cos \theta H_{1}+\sin \theta J H_{2}\right)+\mathbf{R} H_{2} .
$$

We denote by $\left[W_{\lambda}\right] \in \widetilde{G r}_{2}(\mathfrak{p})$ a 2 -dimensional vector subspace $W_{\lambda}$ oriented by the basis $\left\{\cos \theta H_{1}+\sin \theta J H_{2}, H_{2}\right\}$. Then by Lemma 3.4 we obtain

$$
\begin{aligned}
\mu\left(\left[W_{\lambda}\right]\right) & =-\left[\cos \theta H_{1}+\sin \theta J H_{2}, H_{2}\right] \\
& =-\sin \theta\left[J H_{2}, H_{2}\right] \in \mathfrak{c}(\mathfrak{k}) .
\end{aligned}
$$

Hence for each $\lambda \in S^{1}$ the $K$-orbit $K \cdot\left[W_{\lambda}\right]$ in $\widetilde{G r}_{2}(\mathfrak{p})=Q_{n}(\mathbf{C})$ is an isotropic orbit. More precisely, $K \cdot\left[W_{\lambda}\right]$ is a Lagrangian orbit if $\lambda \neq \pm \sqrt{-1}$, and an isotropic orbit of dimension less than $n$ if $\lambda= \pm \sqrt{-1}$. If $(U, K)=(S O(3) \times$ $S O(n+1), S O(2) \times S O(n))(n \geq 2)$, then $\operatorname{dim}\left(K \cdot\left[W_{ \pm \sqrt{-1}}\right]\right)=0$. If $(U, K)=$ $(S O(m+2), S O(2) \times S O(m))(n=2 m-2, m \geq 3)$, then

(1) $\operatorname{dim}\left(K \cdot\left[W_{ \pm \sqrt{-1}}\right]\right)=m-1$ and $K \cdot\left[W_{ \pm \sqrt{-1}}\right]$ is an isotropic orbit diffeomorphic to $S O(m) / S(O(1) \times O(m-1)) \cong \mathbf{R} P^{m-1}$,

(2) for each $\lambda \in S^{1} \backslash\{ \pm \sqrt{-1}\}, \operatorname{dim}\left(K \cdot\left[W_{\lambda}\right]\right)=n=2 m-2$ and $K \cdot\left[W_{\lambda}\right]=$ $K \cdot\left[W_{-\bar{\lambda}}\right]$ is a Lagrangian orbit diffeomorphic to $(S O(2) \times S O(m)) /\left(\mathbf{Z}_{2} \times\right.$ $\left.\mathbf{Z}_{4} \times S O(m-2)\right) \cong N^{n} / \mathbf{Z}_{4}$.

4.3. Classification theorem of Lagrangian orbits in complex hyperquadrics. In the case (a) or (b), we already know all Lagrangian orbits. In the case of (c) or (d), as we showed in Subsection 4.2, there exists an $S^{1}$ family $\left[W_{\lambda}\right] \in \widetilde{\operatorname{Gr}}_{2}(\mathfrak{p})$ with $\lambda \in S^{1}=\{\lambda \in \mathbf{C}|| \lambda \mid=1\}$ satisfying the following conditions :

(1) $\left[W_{1}\right]=[\mathfrak{a}],\left[W_{-\lambda}\right]=-\left[W_{\lambda}\right]$.

(2) $\mu\left(\left[W_{\lambda}\right]\right) \in \mathfrak{c}(\mathfrak{k})$ and $\mu\left(\left[W_{-\lambda}\right]\right)=-\mu\left(\left[W_{\lambda}\right]\right)$.

(3) For each $\lambda \in S^{1}$ with $\lambda \neq \pm \sqrt{-1}$, the $K$-orbit $K \cdot\left[W_{\lambda}\right]$ is a Lagrangian orbit in $Q_{n}(\mathbf{C})$, and the $K$-orbits $K \cdot\left[W_{ \pm \sqrt{-1}}\right]$ are isotropic orbits in 
$Q_{n}(\mathbf{C})$ with $\operatorname{dim} K \cdot\left[W_{ \pm \sqrt{-1}}\right]=0$ in case (c) and $\operatorname{dim} K \cdot\left[W_{ \pm \sqrt{-1}}\right]=$ $m-1$ in case $(\mathrm{d})$.

(4) The square norm $\|\mu\|^{2}$ of the moment map satisfies

$$
\begin{aligned}
& \operatorname{Max}\left\{\left\|\mu\left(\left[W_{\lambda}\right]\right)\right\|^{2} \mid \lambda \in S^{1}\right\} \\
= & \left\|\mu\left(\left[W_{\sqrt{-1}}\right]\right)\right\|^{2}=\left\|\mu\left(\left[W_{-\sqrt{-1}}\right]\right)\right\|^{2} \\
= & \operatorname{Max}\left\{\|\mu([V])\|^{2} \mid[V] \in \widetilde{\mathrm{Gr}} r_{2}(\mathfrak{p}) \text { with } \mu([V]) \in \mathfrak{c}(\mathfrak{k})\right\} .
\end{aligned}
$$

Thus we have

$$
\left\|\mu\left(\left[W_{ \pm 1}\right]\right)\right\|^{2}=\|\mu( \pm[\mathfrak{a}])\|^{2}=0 \leq\left\|\mu\left(\left[V_{0}\right]\right)\right\|^{2} \leq\left\|\mu\left(\left[W_{ \pm \sqrt{-1}}\right]\right)\right\|^{2} .
$$

Since $\operatorname{Im} \mu \cap \mathfrak{c}(\mathfrak{k})$ is a 1-dimensional connected compact subset by Proposition 1.3 (2), there is $\lambda_{0} \in S^{1}$ such that $\left\|\mu\left(\left[V_{0}\right]\right)\right\|^{2}=\left\|\mu\left(\left[W_{\lambda_{0}}\right]\right)\right\|^{2}$. Therefore we obtain $\xi=\mu\left(\left[V_{0}\right]\right)=\mu\left(\left[W_{\lambda_{0}}\right]\right)$ or $\xi=\mu\left(\left[V_{0}\right]\right)=\mu\left(\left[W_{-\lambda_{0}}\right]\right.$. Since $\mu^{-1}(\xi)$ is connected by Proposition $1.3(1)$, we conclude that $K \cdot\left[V_{0}\right]=\mu^{-1}(\xi)=K \cdot\left[W_{\lambda_{0}}\right]$ or $K \cdot\left[V_{0}\right]=\mu^{-1}(\xi)=K \cdot\left[W_{-\lambda_{0}}\right]$.

Therefore our classification of compact homogeneous Lagrangian submanifolds in complex hyperquadrics are described as follows.

Theorem 4.1. Let $L$ be a compact homogeneous Lagrangian submanifold in $Q_{n}(\mathbf{C})$. Then there exists uniquely a homogeneous isoparametric hypersurface $N^{n}$ in $S^{n+1}(1)$ obtained as a principal orbit of the isotropy action of a compact Riemannian symmetric pair $(U, K)$ of rank 2 such that the following statements hold :

(1) If $(U, K)$ is not one of

(a) $\left(S^{1} \times S O(3), S O(2)\right)$,

(b) $(S O(3) \times S O(3), S O(2) \times S O(2))$,

(c) $(S O(3) \times S O(n+1), S O(2) \times S O(n))(n \geq 3)$,

(d) $(S O(m+2), S O(2) \times S O(m))(n=2 m-2, m \geq 3)$,

then $\mathfrak{c}(\mathfrak{k}) \cap \operatorname{Im} \mu=\{0\}$ and

$$
L=\mathcal{G}\left(N^{n}\right) \subset Q_{n}(\mathbf{C}),
$$

which is a minimal Lagrangian submanifold in $Q_{n}(\mathbf{C})$.

(2) If $(U, K)$ is $\left(S^{1} \times S O(3), S O(2)\right)$, then $L$ is a small or great circle in $Q_{1}(\mathbf{C}) \cong S^{2}$.

(3) If $(U, K)$ is $(S O(3) \times S O(3), S O(2) \times S O(2))$, then $L$ is a product of small or great circles of $S^{2}$ in $Q_{2}(\mathbf{C}) \cong S^{2} \times S^{2}$.

(4) If $(U, K)$ is $(S O(3) \times S O(n+1), S O(2) \times S O(n))(n \geq 2)$, then

$$
\operatorname{Im} \mu \cap \mathfrak{c}(\mathfrak{k})=\left\{\mu\left(\left[W_{\lambda}\right]\right) \mid \lambda \in S^{1}\right\}
$$

and

$$
L=K \cdot\left[W_{\lambda}\right] \subset Q_{n}(\mathbf{C}) \quad \text { for some } \lambda \in S^{1} \backslash\{ \pm \sqrt{-1}\},
$$

where $K \cdot\left[W_{\lambda}\right]\left(\lambda \in S^{1}\right)$ is the $S^{1}$-family of Lagrangian or isotropic $K$-orbits satisfying

(a) $K \cdot\left[W_{1}\right]=K \cdot\left[W_{-1}\right]=\mathcal{G}\left(N^{n}\right)$ is a totally geodesic Lagrangian submanifold in $Q_{n}(\mathbf{C})$. 
(b) For each $\lambda \in S^{1} \backslash\{ \pm \sqrt{-1}\}, K \cdot\left[W_{\lambda}\right]$ is a Lagrangian orbit in $Q_{n}(\mathbf{C})$ which is diffeomorphic to $\left(S^{1} \times S^{n-1}\right) / \mathbf{Z}_{2} \cong Q_{2, n}(\mathbf{R})$.

(c) $K \cdot\left[W_{ \pm \sqrt{-1}}\right]$ are isotropic orbits in $Q_{n}(\mathbf{C})$ with $\operatorname{dim} K \cdot\left[W_{ \pm \sqrt{-1}}\right]=$ 0 .

(5) If $(U, K)$ is $(S O(m+2), S O(2) \times S O(m))(n=2 m-2)$, then

$$
\operatorname{Im} \mu \cap \mathfrak{c}(\mathfrak{k})=\left\{\mu\left(\left[W_{\lambda}\right]\right) \mid \lambda \in S^{1}\right\}
$$

and

$$
L=K \cdot\left[W_{\lambda}\right] \subset Q_{n}(\mathbf{C}) \quad \text { for some } \lambda \in S^{1} \backslash\{ \pm \sqrt{-1}\},
$$

where $K \cdot\left[W_{\lambda}\right]\left(\lambda \in S^{1}\right)$ is the $S^{1}$-family of Lagrangian or isotropic orbits satisfying

(a) $K \cdot\left[W_{1}\right]=K \cdot\left[W_{-1}\right]=\mathcal{G}\left(N^{n}\right)$ is a minimal Lagrangian submanifold in $Q_{n}(\mathbf{C})$.

(b) For each $\lambda \in S^{1} \backslash\{ \pm \sqrt{-1}\}, K \cdot\left[W_{\lambda}\right]$ is a Lagrangian orbit in $Q_{n}(\mathbf{C})$, which is diffeomorphic to $(S O(2) \times S O(m)) /\left(\mathbf{Z}_{2} \times \mathbf{Z}_{4} \times\right.$ $S O(m-2))$.

(c) $K \cdot\left[W_{ \pm \sqrt{-1}}\right]$ are isotropic orbits in $Q_{n}(\mathbf{C})$ with $\operatorname{dim} K \cdot\left[W_{ \pm \sqrt{-1}}\right]=$ $m-1$ which is diffeomorphic to $S O(m) / S(O(1) \times O(m-1)) \cong$ $\mathbf{R} P^{m-1}$.

Remark. In each case when $(U, K)=(S O(3) \times S O(n+1), S O(2) \times S O(n))(n \geq$ $3)$ or $(U, K)=(S O(m+2), S O(2) \times S O(m))$ with $n=2 m-2$, there is a nontrivial one-parameter family of Lagrangian orbits in $Q_{n}(\mathbf{C})$. The family contains homogeneous Lagrangian submanifolds which can NEVER be obtained as the Gauss images of homogeneous isoparametric hypersurfaces.

Corollary 4.1. Any compact homogeneous Lagrangian submanifold in a complex hyperquadric is obtained as the Gauss image of a compact homogeneous isoparametric hypersurface in a sphere, or as its Lagrangian deformation.

\section{Hamiltonian Stability of Gauss images of isoparametric HYPERSURFACES IN SPHERES}

Let $N^{n}$ be an oriented compact isoparametric hypersurface embedded in $S^{n+1}(1)$. Now we already know that its Gauss map $\mathcal{G}: N^{n} \rightarrow Q_{n}(\mathbf{C})$ is a minimal Lagrangian immersion. In [37] Palmer showed that the Gauss map $\mathcal{G}: N^{n} \rightarrow Q_{n}(\mathbf{C})$ is Hamiltonian stable if and only if $N^{n}=S^{n} \subset S^{n+1}(1)$ $(g=1)$.

Problem. Investigate the Hamiltonian stability of its Gauss image $\mathcal{G}\left(N^{n}\right)=$ $N^{n} / \mathbf{Z}_{g}$ embedded in $Q_{n}(\mathbf{C})$ as a compact minimal Lagrangian submanifold.

Let $g_{Q_{n}(\mathbf{C})}^{\text {std }}$ denote the $S O(n+2)$-invariant Riemannian metric induced from the standard Euclidean metric of $\mathbf{R}^{n+2}$, whose Einstein constant is equal to $n$. Let $g_{Q_{n}(\mathbf{C})}^{\mathrm{KC}}$ denote the $S O(n+2)$-invariant Riemannian metric induced from the Killing-Cartan form of $S O(n+2)$, whose Einstein constant is equal to $\frac{1}{2}$ (cf. [18]). We also can use Table 1 in Section 1 to determine the Hamiltonian stability in the cases of $g=1$ and $g=2$, because $\mathcal{G}\left(N^{n}\right)$ is a totally geodesic Lagrangian submanifold in $Q_{n}(\mathbf{C})$ in these cases. 
$g=1: \mathcal{G}\left(N^{n}\right)=Q_{1, n+1}(\mathbf{R}) \subset Q_{n}(\mathbf{C})$ is Hamiltonian stable.

$g=2: N^{n}=S^{m_{1}} \times S^{m_{2}}\left(1 \leq m_{1} \leq m_{2}\right)$ are the so called Clifford hypersurfaces.

If $m_{2}-m_{1} \geq 3$, then $\mathcal{G}\left(N^{n}\right)=Q_{m_{1}+1, m_{2}+1}(\mathbf{R}) \subset Q_{n}(\mathbf{C})$ is Not Hamiltonian stable. Otherwise $\mathcal{G}\left(N^{n}\right)=Q_{m_{1}+1, m_{2}+1}(\mathbf{R}) \subset Q_{n}(\mathbf{C})$ is Hamiltonian stable.

In the case of $g=3$, all isoparametric hypersurfaces are homogeneous by E. Cartan's result. In this section we prove the following result.

Theorem 5.1. Suppose that $g=3$, that is, $N^{n}$ is one of the following isoparametric hypersurfaces : (1) $S O(3) / \mathbf{Z}_{2}+\mathbf{Z}_{2}$, (2) $S U(3) / T^{2}$, (3) $S p(3) / S p(1)^{3}$, (4) $F_{4} / \operatorname{Spin}(8)$. Then $L=\mathcal{G}\left(N^{n}\right) \subset Q_{n}(\mathbf{C})$ is strictly Hamiltonian stable.

Remark. In case $g=3$ the induced metrics from $Q_{n}(\mathbf{C})$ have nice intrinsic properties. In (1), $L$ has constant sectional curvature 1/96 if the Einstein constant of $Q_{n}(\mathbf{C})$ is equal to $\frac{1}{2}$. In (1)-(4), $L$ has non-negative sectional curvatures. In fact, the induced invariant metrics are normal homogenous metrics.

Refer also [24, 19], 23] for investigation on the first eigenvalue of the Laplacian of homogeneous isoparametric hypersurfaces in spheres.

We recall some results from the spherical function theory on compact homogeneous spaces to order to determine the first eigenvalues of $\mathcal{G}\left(N^{n}\right)$ relative to the induced metric from $Q_{n}(\mathbf{C})$,

Suppose that $\langle,\rangle_{\mathfrak{k}}$ is an $\mathrm{Ad} K$-invariant inner product of $\mathfrak{k}$. For a compact Lie subgroup $S$ of $K$ with Lie algebra $\mathfrak{s}$, we take the orthogonal direct sum decomposition $\mathfrak{k}=\mathfrak{s}+\mathfrak{m}$ and the vector space $\mathfrak{m}$ is identified with the tangent vector space $T_{e S}(K / S)$ of compact homogeneous space $K / S$ at the origin $e S$. The Casimir operator $\mathcal{C}$ of $(K, S)$ with respect to $\langle,\rangle_{\mathfrak{k}}$ by $\mathcal{C}:=\sum_{i=1}^{n} X_{i}$, where $\left\{X_{i} \mid i=1, \cdots, n\right\}$ is an orthonormal basis of $\mathfrak{m}$ with respect to $\langle,\rangle_{\mathfrak{k}}$. Let $\mathcal{D}(K)$ be the complete set of all inequivalent irreducible unitary representations of a compact Lie group $K$. For a maximal abelian subalgebra $\mathfrak{h}$ of $\mathfrak{k}$, let $\Sigma(K)$ be the set of all roots of $\mathfrak{k}$ with respect to $\mathfrak{h}$ and let $\Sigma^{+}(K)$ be its subset of all positive $\alpha \in \Sigma(K)$ relative to a linear order on $\mathfrak{h}$. Set

$$
\begin{aligned}
\Gamma(K) & :=\{\xi \in \mathfrak{h} \mid \exp (\xi)=e\}, \\
Z(K) & :=\left\{\Lambda \in \mathfrak{h}^{*} \mid \Lambda(\xi) \in \mathbf{Z}\right\}, \\
D(K) & :=\left\{\Lambda \in \mathfrak{h}^{*} \mid(\Lambda, \alpha) \geq 0 \text { for each } \alpha \in \Sigma^{+}(K)\right\} .
\end{aligned}
$$

Then we know that there is a bijective correspondence between $D(K)$ and $\mathcal{D}(K)$ : Each $\Lambda \in D(K)$ uniquely corresponds to an irreducible unitary representation $\left(V_{\Lambda}, \rho_{\Lambda}\right)$ of $K$ with the highest weight $\Lambda$, up to the equivalence. Here we denote by $\langle,\rangle_{V_{\Lambda}}$ a $K$-invariant Hermitian inner product equipped on $V_{\Lambda}$. Let $\left(V_{\Lambda}\right)_{S}$ denote the vector subspace of $V_{\Lambda}$ consisting of all vectors fixed by $\rho_{\Lambda}(S)$. Define

$$
D(K, S):=\left\{\Lambda \in D(K) \mid\left(V_{\Lambda}\right)_{S} \neq\{0\}\right\} .
$$

By the Peter-Weyl's theorem we know that

$$
C^{\infty}(K / S)=\bigoplus_{\Lambda \in D(K, S)}\left(V_{\Lambda}\right)_{S}^{*} \otimes V_{\Lambda}
$$


Here each $w \in\left(V_{\Lambda}\right)_{S}$ and each $v \in V_{\Lambda}$ correspond to $f \in C^{\infty}(K / S)$ defined by

$$
f(a S):=\left\langle\rho_{\Lambda}(a) w, v\right\rangle_{\Lambda} \quad(a K \in K / S) .
$$

Then the Laplace-Beltrami operator $\Delta_{K / S}$ with respect to the metric $g_{K / S}$ on $K / S$ induced by $\langle,\rangle_{\mathfrak{k}}$ is expressed in terms of $\mathcal{C}$ as

$$
\left(\Delta_{K / S} f\right)(a S)=\left\langle\rho_{\Lambda}(a)\left(\left(d \rho_{\Lambda}(\mathcal{C}) w\right)\right), v\right\rangle_{\Lambda} .
$$

By Schur's lemma there is a real constant $c\left(\Lambda,\langle,\rangle_{\mathfrak{k}}\right) \leq 0$ such that

$$
\left(d \rho_{\Lambda}(\mathcal{C})\right) v=\sum_{i=1}^{n}\left(d \rho_{\Lambda}\left(X_{\gamma, i}\right)\right)^{2} v=c\left(\Lambda,\langle,\rangle_{\mathfrak{k}}\right) v \quad \text { for each } v \in V_{\lambda} .
$$

The eigenvalue $c\left(\Lambda,\langle,\rangle_{\mathfrak{k}}\right)$ is described by the Freudenthal's formula

$$
c\left(\Lambda,\langle,\rangle_{\mathfrak{k}}\right)=-\langle\Lambda, \Lambda+2 \delta\rangle_{\mathfrak{k}}
$$

where $2 \delta=\sum_{\alpha \in \Sigma^{+}(K)} \alpha$.

We shall consider our compact homogeneous spaces $K / K_{0}$ and $K / K_{[\mathfrak{a}]}$. The tangent vector spaces $T_{e K_{0}}\left(K / K_{0}\right)$ and $T_{e K_{[\mathfrak{a}]}}\left(K / K_{[\mathfrak{a}]}\right)$ can be identified with the vector subspace $\mathfrak{m}$ in (3.6) of $\mathfrak{k}$. Let $\langle$,$\rangle denote the K_{\mathfrak{a}}$-invariant inner product of $\mathfrak{m}$ corresponding to the $K$-invariant Riemannian metric $\mathcal{G}^{*} g_{Q_{n}(\mathbf{C})}^{\text {std }}$ on $K / K_{0}$ induced from $g_{Q_{n}(\mathbf{C})}^{\text {std }}$ through the Gauss map $\mathcal{G}$. Using the standard basis (3.7), (3.8), we compute

$$
\left[(d \mathcal{G})_{e K_{0}}\left(X_{\gamma, i}\right)\right](H)=\pi_{\mathfrak{a}^{\perp}}\left(\left[X_{\gamma, i}, H\right]\right)=\left[X_{\gamma, i}, H\right]=-\sqrt{-1} \gamma(H) Y_{\gamma, i}
$$

and

$$
\left\langle(d \mathcal{G})_{e K_{0}}\left(X_{\gamma, i}\right),(d \mathcal{G})_{e K_{0}}\left(X_{\gamma^{\prime}, j}\right)\right\rangle_{\mathfrak{u}}=\|\gamma\|_{\mathfrak{u}}^{2}\left\langle X_{\gamma, i}, X_{\gamma^{\prime}, j}\right\rangle_{\mathfrak{u}}
$$

Thus we see that

$$
\left\{\frac{1}{\|\gamma\|_{\mathfrak{u}}} X_{\gamma, i} \mid \gamma \in \Sigma^{+}(U, K), i=1,2, \cdots, m(\gamma)\right\}
$$

is an orthonormal basis of $\mathfrak{m}$ with respect to $\langle$,$\rangle .$

Suppose that $g=3$. Since $(U, K)$ is type $A_{2}$, we have

$$
\langle,\rangle=\left\|\gamma_{1}\right\|_{\mathfrak{u}}^{2}\langle,\rangle_{\mathfrak{u}}
$$

on $\mathfrak{m}$. On the other hand, since $K$ is simple, we can define an $\operatorname{Ad} K$-invariant inner product $\langle,\rangle_{\mathfrak{k}}=-B_{\mathfrak{k}}($,$) of \mathfrak{k}$ by using the Killing-Cartan form $B_{\mathfrak{k}}$ of $\mathfrak{k}$ and moreover there is $b>0$ such that

$$
\langle,\rangle_{\mathfrak{k}}=b\langle,\rangle_{\mathfrak{u}} .
$$

on $\mathfrak{k}$. Set $C:=\left\|\gamma_{1}\right\|_{\mathfrak{u}}^{2} \cdot b^{-1}$. Then we have

$$
\langle,\rangle=C\langle,\rangle_{\mathfrak{k}}
$$

Thus we obtain

Lemma 5.1. $L=\mathcal{G}\left(N^{n}\right) \subset Q_{n}(\mathbf{C})$ is Hamiltonian stable if and only if

$$
\operatorname{Min}\left\{-c\left(\Lambda,\langle,\rangle_{\mathfrak{k}}\right) \mid 0 \neq \Lambda \in D\left(K, K_{[\mathfrak{a}]}\right)\right\}
$$

is equal to $\mathrm{Cn}$. 
Lemma 5.2. The constants $b^{-1},\left\|\gamma_{1}\right\|_{\mathfrak{u}}^{2}, C n$ in each case are given as in the following table:

\begin{tabular}{|c|c|c|c|c|c|c|}
\hline$(U, K)$ & $n$ & $\operatorname{dim} \mathfrak{p}$ & $\operatorname{dim} \mathfrak{k}$ & $b^{-1}$ & $\left\|\gamma_{1}\right\|_{\mathfrak{u}}^{2}$ & $C n$ \\
\hline$(S U(3), S O(3))$ & 3 & 5 & 3 & 6 & $1 / 3$ & 6 \\
\hline$(S U(3) \times S U(3), S U(3))$ & 6 & 8 & 8 & 2 & $1 / 6$ & 2 \\
\hline$(S U(6), S p(3))$ & 12 & 14 & 21 & $3 / 2$ & $1 / 12$ & $3 / 2$ \\
\hline$\left(E_{6}, F_{4}\right)$ & 24 & 26 & 52 & $4 / 3$ & $1 / 24$ & $4 / 3$ \\
\hline
\end{tabular}

Proof. Choose an orthonormal basis $\left\{e_{j}\right\}$ of $\mathfrak{m}$ and an orthonormal basis $\left\{H_{\nu} \mid \nu=\right.$ $1,2\}$ of $\mathfrak{a}$ with respect to $\langle,\rangle_{\mathfrak{u}}$. Then we compute

$$
\begin{gathered}
\sum_{j}\left\langle e_{j}, e_{j}\right\rangle=C b \sum_{j}\left\langle e_{j}, e_{j}\right\rangle_{\mathfrak{u}}=C b \operatorname{dim} \mathfrak{m}, \\
\sum_{j}\left\langle e_{j}, e_{j}\right\rangle=\sum_{\nu} \sum_{j}\left\langle\left[e_{j}, H_{\nu}\right],\left[e_{j}, H_{\nu}\right]\right\rangle_{\mathfrak{u}}=-\sum_{\nu}\left\langle\sum_{j}\left(\operatorname{ad} e_{j}\right)^{2} H_{\nu}, H_{\nu}\right\rangle_{\mathfrak{u}} \\
=-b \sum_{\nu}\left\langle\sum_{j}\left(\operatorname{ad} e_{j} / \sqrt{b}\right)^{2} H_{\nu}, H_{\nu}\right\rangle_{\mathfrak{u}}=-2 b \cdot c\left(\mathfrak{k}, \operatorname{ad}_{\mathfrak{p}},\langle,\rangle_{\mathfrak{k}}\right),
\end{gathered}
$$

and

$$
\begin{aligned}
\sum_{j}\left\langle e_{j}, e_{j}\right\rangle & =-\sum_{\nu} \sum_{j}\left\langle\left(\operatorname{ad} H_{\nu}\right)^{2} e_{j}, e_{j}\right\rangle_{\mathfrak{u}}=-\sum_{\nu} \operatorname{tr}_{\mathfrak{k}}\left(\operatorname{ad} H_{\nu}\right)^{2} \\
& =-\frac{1}{2} \sum_{\nu} \operatorname{tr}_{\mathfrak{u}}\left(\operatorname{ad} H_{\nu}\right)^{2}=\frac{1}{2} \sum_{\nu}\left\langle H_{\nu}, H_{\nu}\right\rangle_{\mathfrak{u}}=1
\end{aligned}
$$

Thus we have

$$
C b \operatorname{dim} \mathfrak{m}=-2 b \cdot c\left(\mathfrak{k}, \operatorname{ad}_{\mathfrak{p}},\langle,\rangle_{\mathfrak{k}}\right)=1
$$

Since

$$
b=\frac{\operatorname{dim} \mathfrak{k}}{\operatorname{dim} \mathfrak{k}-(\operatorname{dim} \mathfrak{p}) \cdot c\left(\mathfrak{k}, \operatorname{ad}_{\mathfrak{p}},\langle,\rangle_{\mathfrak{k}}\right)}
$$

by [1, p. 591, Proposition 2.2], we obtain

$$
\begin{aligned}
b & =1-\frac{1}{2} \frac{\operatorname{dim} \mathfrak{p}}{\operatorname{dim} \mathfrak{k}}, \\
-c\left(\mathfrak{k}, \operatorname{ad}_{\mathfrak{p}},\langle,\rangle_{\mathfrak{k}}\right) & =\frac{\operatorname{dim\mathfrak {k}}}{2 \operatorname{dim} \mathfrak{k}-\operatorname{dim} \mathfrak{p}}, \\
\left\|\gamma_{1}\right\|_{\mathfrak{u}}^{2} & =C b=\frac{1}{\operatorname{dim} \mathfrak{m}}, \\
C & =\frac{2 \operatorname{dim} \mathfrak{k}}{(\operatorname{dim} \mathfrak{m})(2 \operatorname{dim} \mathfrak{k}-\operatorname{dim} \mathfrak{p})} .
\end{aligned}
$$

(1) The case $(U, K)=(S U(3), S O(3))$ :

Define a basis $\left\{E_{1}, E_{2}, E_{3}\right\}$ of Lie algebra $\mathfrak{s u}(2)$ of $S U(2)$ by

$$
E_{1}:=\left(\begin{array}{cc}
\sqrt{-1} & 0 \\
0 & -\sqrt{-1}
\end{array}\right), E_{2}:=\left(\begin{array}{cc}
0 & 1 \\
-1 & 0
\end{array}\right), E_{3}:=\left(\begin{array}{cc}
0 & \sqrt{-1} \\
\sqrt{-1} & 0
\end{array}\right) .
$$


Let $\psi: S U(2) \rightarrow S O(3)$ be a universal covering Lie group homomorphism defined by

$$
\operatorname{Ad}(a)\left(E_{1}, E_{2}, E_{3}\right)=\left(E_{1}, E_{2}, E_{3}\right) \psi(a) .
$$

Set $\tilde{K}:=S U(2), \tilde{K}_{[\mathfrak{a}]}:=\psi^{-1}\left(K_{[\mathfrak{a}]}\right)$ and $\tilde{K}_{0}:=\psi^{-1}\left(K_{0}\right)$. Then we have $\tilde{K} / \tilde{K}_{[\mathfrak{a}]} \cong K / K_{[\mathfrak{a}]}$ and $\tilde{K} / \tilde{K}_{0} \cong K / K_{0}$. Explicitly the group $K_{0}$ is a finite subgroup of order 4 generated by

$$
\left(\begin{array}{lll}
1 & 0 & 0 \\
0 & 1 & 0 \\
0 & 0 & 1
\end{array}\right),\left(\begin{array}{ccc}
1 & 0 & 0 \\
0 & -1 & 0 \\
0 & 0 & -1
\end{array}\right),\left(\begin{array}{ccc}
-1 & 0 & 0 \\
0 & -1 & 0 \\
0 & 0 & 1
\end{array}\right),\left(\begin{array}{ccc}
-1 & 0 & 0 \\
0 & 1 & 0 \\
0 & 0 & -1
\end{array}\right)
$$

and $K_{[\mathfrak{a}]}$ is a finite subgroup of order 12 generated by $K_{0}$ and

$$
\left(\begin{array}{lll}
0 & 0 & 1 \\
1 & 0 & 0 \\
0 & 1 & 0
\end{array}\right),\left(\begin{array}{lll}
0 & 1 & 0 \\
0 & 0 & 1 \\
1 & 0 & 0
\end{array}\right)=\left(\begin{array}{lll}
0 & 0 & 1 \\
1 & 0 & 0 \\
0 & 1 & 0
\end{array}\right)^{2}
$$

The group $\tilde{K}_{0}$ is a finite subgroup of order 8 generated by

$$
\pm\left(\begin{array}{ll}
1 & 0 \\
0 & 1
\end{array}\right), \pm\left(\begin{array}{cc}
0 & -1 \\
1 & 0
\end{array}\right), \pm\left(\begin{array}{cc}
\sqrt{-1} & 0 \\
0 & -\sqrt{-1}
\end{array}\right), \pm\left(\begin{array}{cc}
0 & \sqrt{-1} \\
\sqrt{-1} & 0
\end{array}\right)
$$

The group $\tilde{K}_{[\mathfrak{a}]}$ is a finite subgroup of order 24 generated by $\tilde{K}_{[\mathfrak{a}]}$ and

$\pm\left(\begin{array}{cc}\frac{1+\sqrt{-1}}{2} & \frac{1+\sqrt{-1}}{2} \\ \frac{-1+\sqrt{-1}}{2} & \frac{1-\sqrt{-1}}{2}\end{array}\right), \pm\left(\begin{array}{cc}\frac{-1+\sqrt{-1}}{2} & \frac{1+\sqrt{-1}}{2} \\ \frac{-1+\sqrt{-1}}{2} & \frac{-1-\sqrt{-1}}{2}\end{array}\right)= \pm\left(\begin{array}{cc}\frac{1+\sqrt{-1}}{2} & \frac{1+\sqrt{-1}}{2} \\ \frac{-1+\sqrt{-1}}{2} & \frac{1-\sqrt{-1}}{2}\end{array}\right)^{2}$.

We know that

$$
\mathcal{D}(S U(2))=\left\{\left(V_{m}, \rho_{m}\right) \mid m \in \mathbf{Z}, m \geq 0\right\}
$$

Here $V_{m}$ denotes the complex vector space of complex homogeneous polynomials of degree $m$ with two variables $z_{0}, z_{1}$ and the representation $\rho_{m}$ of $S U(2)$ on $V_{m}$ is defined by

$$
\left(\rho_{n}\left(\begin{array}{cc}
a & -\bar{b} \\
b & \bar{a}
\end{array}\right) f\right)\left(z_{0}, z_{1}\right)=f\left(\left(z_{0}, z_{1}\right)\left(\begin{array}{cc}
a & -\bar{b} \\
b & \bar{a}
\end{array}\right)\right) \quad \text { for each }\left(\begin{array}{cc}
a & -\bar{b} \\
b & \bar{a}
\end{array}\right) \in S U(2) \text {. }
$$

Set

$$
v_{k}^{(m)}:=\frac{1}{\sqrt{k !(m-k) !}} z_{0}^{m-k} z_{1}^{k} \in V_{m} \quad(k=0,1, \ldots, m)
$$

and the standard Hermitian inner product $\langle\langle\rangle$,$\rangle of V_{m}$ invariant under $\rho_{m}$ is defined such that $\left\{v_{0}^{(m)}, \ldots, v_{m}^{(m)}\right\}$ is a unitary basis of $V_{m}$. Then there is a bijective correspondence between $\mathcal{D}(S U(2))$ and $D(S U(2))=\left\{m \Lambda_{1} \mid m \in\right.$ $\mathbf{Z}, m \geq 0\}$, where $\Lambda_{1}$ denotes the fundamental weight of $\mathfrak{s u}(2)$.

The Killing-Cartan form is given by

$$
B_{\mathfrak{s u}(2)}(X, Y)=4 \operatorname{tr}(X Y) \text { for each } X, Y \in \mathfrak{s u}(2) .
$$

Let $\left\{X_{1}, X_{2}, X_{3}\right\}$ be an orthonormal basis of $\tilde{\mathfrak{k}}=\mathfrak{s u}(2)$ with respect to $\langle,\rangle_{\tilde{\mathfrak{k}}}$ defined by

$$
X_{1}=\frac{1}{2 \sqrt{2}} E_{1}, \quad X_{2}=\frac{1}{2 \sqrt{2}} E_{2}, \quad X_{3}=\frac{1}{2 \sqrt{2}} E_{3} .
$$


The Casimir operator $\mathcal{C}$ of $S U(2)$ with respect to the inner product $\langle,\rangle_{\tilde{\mathfrak{k}}}$ is given as

$$
\mathcal{C}=\sum_{i=1}^{3}\left(X_{i}\right)^{2}=\frac{1}{8} \sum_{i=1}^{3}\left(E_{i}\right)^{2}
$$

Then we obtain

Lemma 5.3 (cf. [24]). tor $\mathcal{C}$ is given as

(1) The eigenvalue formula for the Casimir opera-

$$
\rho_{m}(\mathcal{C}) v=-\frac{m(m+2)}{8} v
$$

for each $v \in V_{m}$.

(2) $\mathcal{D}\left(\tilde{K}, \tilde{K}_{0}\right)$ is determined as follows : $\left(\rho_{m}, V_{m}\right) \in \mathcal{D}\left(\tilde{K}, \tilde{K}_{0}\right)$ if and only if $m$ is even and $m \geq 4$. Then for each $\left(\rho_{m}, V_{m}\right) \in \mathcal{D}\left(\tilde{K}, \tilde{K}_{0}\right)$ with $m=2 p$ for some integer $p$, the vector subspace $\left(V_{m}\right)_{\tilde{K}_{0}}$ is spanned by

$$
w_{i}:=\frac{1}{2}\left(v_{2(i-1)}^{(m)}+v_{4 l-2(i-1)}^{(m)}\right)(i=1, \cdots, \ell+1) \quad \text { if } p=2 \ell,
$$

or

$$
w_{i}^{\prime}:=\frac{1}{2}\left(v_{2 i-1}^{(m)}-v_{4 \ell-2 i+3)}^{(m)}\right)(i=1, \cdots, \ell) \quad \text { if } p=2 \ell+1 .
$$

We must examine eigenvalues of $\mathcal{C}$ smaller than or equal to 6 for $\left(\rho_{m}, V_{m}\right) \in$ $\mathcal{D}\left(\tilde{K}, \tilde{K}_{[\mathfrak{a}]}\right) \subset \mathcal{D}\left(\tilde{K}, \tilde{K}_{0}\right)$. We observe that all the eigenvalues of $\mathcal{C}$ smaller than or equal to 6 for $\left(\rho_{m}, V_{m}\right) \in \mathcal{D}\left(\tilde{K}, \tilde{K}_{0}\right)$ are $3(m=4)$ and $6(m=6)$.

For $m=4$, we shall show that $\left(\rho_{4}, V_{4}\right) \notin \mathcal{D}\left(\tilde{K}, \tilde{K}_{[\mathfrak{a}]}\right)(m=4)$. If we set

$$
B=\left(\begin{array}{cc}
\frac{1+\sqrt{-1}}{2} & \frac{1+\sqrt{-1}}{2} \\
\frac{-1+\sqrt{-1}}{2} & \frac{1-\sqrt{-1}}{2}
\end{array}\right) \in \tilde{K}_{[\mathfrak{a}]},
$$

then $B \notin \tilde{K}_{0}$ and

$$
\begin{aligned}
& \left(\rho_{4}(B)\right) w_{1}=-\frac{1}{4 \sqrt{4 !}}\left(z_{0}^{4}-6 z_{0}^{2} z_{1}^{2}+z_{1}^{4}\right) \\
& \left(\rho_{4}(B)\right) w_{2}=-\frac{1}{4 \cdot 2 !}\left(z_{0}^{4}+2 z_{0}^{2} z_{1}^{2}+z_{1}^{4}\right) .
\end{aligned}
$$

Thus if we assume that $w=a w_{1}+b w_{2} \in\left(V_{m}\right)_{\tilde{K}_{[\mathfrak{a}]}}$, then by the above equations $\left(\rho_{4}(B)\right) w=w$ implies that $w=0$. Hence we obtain $\left(V_{4}\right)_{\tilde{K}_{[\mathfrak{a}]}}=\{0\}$.

For $m=6$, since a simple computation implies that $\left(\rho_{6}(B)\right) w_{1}^{\prime}=\frac{1}{2} w_{1}^{\prime}$, we obtain $\left(V_{6}\right)_{\tilde{K}_{[\mathfrak{a}]}}=\operatorname{span}_{\mathbf{C}}\left\{w_{1}^{\prime}\right\}$ and thus $\operatorname{dim}\left(V_{6}\right)_{\tilde{K}_{[\mathfrak{a}]}}=1$.

We conclude that $L=\mathcal{G}\left(N^{3}\right)$ is Hamiltonian stable and the nullity is equal to $7=\operatorname{dim}(S O(5))-3$, and hence $L=\mathcal{G}\left(S O(3) / \mathbf{Z}_{2}+\mathbf{Z}_{2}\right)$ is strictly Hamiltonian stable.

(2) The case $(U, K)=(S U(3) \times S U(3), S U(3))$ : Then

$$
\begin{aligned}
& K=S U(3), K_{0}=T^{2} \\
& D\left(K, K_{0}\right)=D\left(S U(3), T^{2}\right), D\left(K, K_{[\mathfrak{a}]}\right)=D\left(S U(3), T^{2} \cdot \mathbf{Z}_{3}\right)
\end{aligned}
$$


and

$$
D\left(S U(3), T^{2} \cdot \mathbf{Z}_{3}\right) \subset D\left(S U(3), T^{2}\right) .
$$

Let $\left\{\alpha_{1}, \alpha_{2}\right\}$ be the fundamental root system of $S U(3)$ and $\left\{\Lambda_{1}, \Lambda_{2}\right\}$ be the fundamental weight system of $S U(3)$. We use the results of Satoru Yamaguchi [41] as follows : Each $\Lambda \in D\left(S U(3), T^{2}\right)$ can be uniquely expressed as

$$
\Lambda=\sum_{i} m_{i} \Lambda_{i}=\sum_{i} p_{i} \alpha_{i}
$$

where $m_{i} \in \mathbf{Z}, m_{i} \geq 0, p_{i} \in \mathbf{Z}, p_{i} \geq 1$ and

$$
m_{1}=2 p_{1}-p_{2} \geq 0, \quad m_{2}=-p_{1}+2 p_{2} \geq 0 \text {. }
$$

The eigenvalue formula is

$$
-c\left(\Lambda,\langle,\rangle_{\mathfrak{k}}\right)=\frac{1}{6}\left(m_{1} p_{1}+m_{2} p_{2}+2 p_{1}+2 p_{2}\right)
$$

for each $\Lambda \in D\left(S U(3), T^{2}\right)$. Therefore we get

$$
\begin{aligned}
& \left\{\Lambda \in D\left(S U(3), T^{2}\right) \mid-c\left(\Lambda,\langle,\rangle_{\mathfrak{k}}\right) \leq 2\right\} \\
= & \left\{0,3 \Lambda_{1}\left(\left(p_{1}, p_{2}\right)=(2,1)\right), 3 \Lambda_{2}\left(\left(p_{1}, p_{2}\right)=(1,2)\right),\right. \\
& \left.\Lambda_{1}+\Lambda_{2}\left(\left(p_{1}, p_{2}\right)=(1,1)\right)\right\} .
\end{aligned}
$$

Since $\Lambda_{1}+\Lambda_{2} \notin D\left(S U(3), T^{2} \cdot \mathbf{Z}_{3}\right)$, we obtain that

$$
\begin{aligned}
& \left\{\Lambda \in D\left(S U(3), T^{2} \cdot \mathbf{Z}_{3}\right) \mid-c\left(\Lambda,\langle,\rangle_{\mathfrak{k}}\right) \leq 2\right\} \\
= & \left\{0,3 \Lambda_{1}\left(\left(p_{1}, p_{2}\right)=(2,1)\right), 3 \Lambda_{2}\left(\left(p_{1}, p_{2}\right)=(1,2)\right)\right\} .
\end{aligned}
$$

and $-c\left(3 \Lambda_{1},\langle,\rangle_{\mathfrak{k}}\right)=-c\left(3 \Lambda_{2},\langle,\rangle_{\mathfrak{k}}\right)=2$. By [20] we have $\operatorname{dim}_{\mathbf{C}}\left(V_{3 \Lambda_{1}}\right)_{T^{2}}=$ $\operatorname{dim}_{\mathbf{C}}\left(V_{3 \Lambda_{2}}\right)_{T^{2}}=1$. Hence the nullity is equal to $\operatorname{dim}\left(V_{3 \Lambda_{1}}\right)+\operatorname{dim}\left(V_{3 \Lambda_{2}}\right)=$ $10+10=20=\operatorname{dim}(S O(8))-\operatorname{dim}(S U(3))$. We conclude that $L=\mathcal{G}\left(S U(3) / T^{2}\right)$ is strictly Hamiltonian stable.

(3) The case $(U, K)=(S U(6), S p(3))$ : Then

$$
\begin{aligned}
& K=S p(3), K_{0}=S p(1)^{3}, \\
& D\left(K, K_{0}\right)=D\left(S p(3), S p(1)^{3}\right), D\left(K, K_{[\mathfrak{a}]}\right)=D\left(S p(3), S p(1)^{3} \cdot \mathbf{Z}_{3}\right)
\end{aligned}
$$

and

$$
D\left(S p(3), S p(1)^{3} \cdot \mathbf{Z}_{3}\right) \subset D\left(S p(3), S p(1)^{3}\right) \subset D\left(S p(3), T^{3}\right) .
$$

Let $\left\{\alpha_{1}, \alpha_{2}, \alpha_{3}\right\}$ be the fundamental root system of $S p(3)$ and $\left\{\Lambda_{1}, \Lambda_{2}, \Lambda_{3}\right\}$ be the fundamental weight system of $S p(3)$. The results from [41] are as follows : Each $\Lambda \in D\left(S p(3), T^{3}\right)$ can be uniquely expressed as

$$
\Lambda=\sum_{i} m_{i} \Lambda_{i}=\sum_{i} p_{i} \alpha_{i}
$$

where $m_{i} \in \mathbf{Z}, m_{i} \geq 0, p_{i} \in \mathbf{Z}, p_{i} \geq 1$ and

$$
m_{1}=2 p_{1}-p_{2} \geq 0, m_{2}=-p_{1}+2 p_{2}-p_{3} \geq 0, m_{3}=-p_{2}+2 p_{3} \geq 0 .
$$

The eigenvalue formula is

$$
-c\left(\Lambda,\langle,\rangle_{\mathfrak{k}}\right)=\frac{1}{16}\left(m_{1} p_{1}+m_{2} p_{2}+2 m_{3} p_{3}+2 p_{1}+2 p_{2}+4 p_{3}\right)
$$


for each $\Lambda \in D\left(S p(3), T^{3}\right)$. Therefore we get

$$
\begin{aligned}
& \left\{\Lambda \in D\left(S p(3), T^{3}\right) \mid-c\left(\Lambda,\langle,\rangle_{\mathfrak{k}}\right) \leq 3 / 2\right\} \\
= & \left\{0,2 \Lambda_{1}\left(\left(p_{1}, p_{2}, p_{3}\right)=(2,2,1)\right),\right. \\
& \left.\Lambda_{2}\left(\left(p_{1}, p_{2}, p_{3}\right)=(1,2,1)\right), \Lambda_{1}+\Lambda_{3}\left(\left(p_{1}, p_{2}, p_{3}\right)=(2,3,2)\right)\right\} .
\end{aligned}
$$

Since we see that $2 \Lambda_{1} \notin D\left(S p(3), S p(1)^{3}\right)$ by using [20], we obtain that

$$
\left\{\Lambda \in D\left(S p(3), S p(1)^{3} \cdot \mathbf{Z}_{3}\right) \mid-c\left(\Lambda,\langle,\rangle_{\mathfrak{k}}\right) \leq 3 / 2\right\}=\left\{0, \Lambda_{1}+\Lambda_{3}\right\} .
$$

and $-c\left(\Lambda_{1}+\Lambda_{3},\langle,\rangle_{\mathfrak{k}}\right)=3 / 2$. By [20] we have $\operatorname{dim}_{\mathbf{C}}\left(V_{\Lambda_{1}+\Lambda_{3}}\right)_{S p(1)^{3}}=1$. Hence the nullity is equal to $\operatorname{dim}\left(\Lambda_{1}+\Lambda_{3}\right)=70=\operatorname{dim}(S O(14))-\operatorname{dim}(S p(3))(=$ $91-21)$. We conclude that $L=\mathcal{G}\left(S p(3) / S p(1)^{3}\right)$ is strictly Hamiltonian stable.

(4) The case $(U, K)=\left(E_{6}, F_{4}\right)$ : Then

$$
\begin{aligned}
& K=F_{4}, K_{0}=\operatorname{Spin}(8), \\
& D\left(K, K_{0}\right)=D\left(F_{4}, \operatorname{Spin}(8)\right), D\left(K, K_{[\mathfrak{a}]}\right)=D\left(F_{4}, \operatorname{Spin}(8) \cdot \mathbf{Z}_{3}\right)
\end{aligned}
$$

and

$$
D\left(F_{4}, \operatorname{Spin}(8) \cdot \mathbf{Z}_{3}\right) \subset D\left(F_{4}, \operatorname{Spin}(8)\right) \subset D\left(F_{4}, T^{4}\right) .
$$

Let $\left\{\alpha_{1}, \alpha_{2}, \alpha_{3}, \alpha_{4}\right\}$ be the fundamental root system of $F_{4}$ and $\left\{\Lambda_{1}, \Lambda_{2}, \Lambda_{3}, \Lambda_{4}\right\}$ be the fundamental weight system of $F_{4}$. The results from [41] are as follows : Each $\Lambda \in D\left(F_{4}, T^{4}\right)$ can be uniquely expressed as

$$
\Lambda=\sum_{i} m_{i} \Lambda_{i}=\sum_{i} p_{i} \alpha_{i}
$$

where $m_{i} \in \mathbf{Z}, m_{i} \geq 0, p_{i} \in \mathbf{Z}, p_{i} \geq 1$ and

$$
\begin{aligned}
& m_{1}=2 p_{1}-p_{2} \geq 0, m_{2}=-p_{1}+2 p_{2}-p_{3} \geq 0, \\
& m_{3}=-2 p_{2}+2 p_{3}-p_{4} \geq 0, m_{4}=-p_{3}+2 p_{4} \geq 0 .
\end{aligned}
$$

The eigenvalue formula is

$$
-c\left(\Lambda,\langle,\rangle_{\mathfrak{k}}\right)=\frac{1}{18}\left(m_{1} p_{1}+m_{2} p_{2}+\frac{1}{2} m_{3} p_{3}+\frac{1}{2} m_{4} p_{4}+2 p_{1}+2 p_{2}+p_{3}+p_{4}\right)
$$

for each $\Lambda \in D\left(F_{4}, T^{4}\right)$. Therefore we get

$$
\begin{aligned}
& \left\{\Lambda \in D\left(F_{4}, T^{4}\right) \mid-c\left(\Lambda,\langle,\rangle_{\mathfrak{k}}\right) \leq 4 / 3\right\} \\
= & \left\{0, \Lambda_{1}\left(\left(p_{1}, p_{2}, p_{3}, p_{4}\right)=(2,3,4,2)\right),\right. \\
& \left.\Lambda_{3}\left(\left(p_{1}, p_{2}, p_{3}, p_{4}\right)=(2,4,6,3)\right), \Lambda_{4}\left(\left(p_{1}, p_{2}, p_{3}, p_{4}\right)=(1,2,3,2)\right)\right\} .
\end{aligned}
$$

Since $\Lambda_{4} \notin D\left(F_{4}, \operatorname{Spin}(8) \cdot \mathbf{Z}_{3}\right)$ and we see $\Lambda_{1} \notin D\left(F_{4}, \operatorname{Spin}(8)\right)$ by using [20] we obtain that

$$
\begin{aligned}
& \left\{\Lambda \in D\left(F_{4}, \operatorname{Spin}(8) \cdot \mathbf{Z}_{3}\right) \mid-c\left(\Lambda,\langle,\rangle_{\mathfrak{k}}\right) \leq 4 / 3\right\} \\
= & \left\{0, \Lambda_{3}\left(\left(p_{1}, p_{2}, p_{3}, p_{4}\right)=(2,4,6,3)\right)\right\} .
\end{aligned}
$$

and $-c\left(\Lambda_{3},\langle,\rangle_{\mathfrak{k}}\right)=4 / 3$. By [20] we have $\operatorname{dim}_{\mathbf{C}}\left(V_{\Lambda_{3}}\right)_{\operatorname{Spin}(8)}=1$. Hence the nullity is equal to $\operatorname{dim}\left(\Lambda_{3}\right)=273=\operatorname{dim}(S O(26))-\operatorname{dim}\left(F_{4}\right)(=325-52)$. We conclude that $L=\mathcal{G}\left(F_{4} / \operatorname{Spin}(8)\right)$ is strictly Hamiltonian stable. This proves the theorem. 


\section{REFERENCES}

[1] A. Amarzaya and Y. Ohnita, Hamiltonian stability of certain minimal Lagrangian submanifolds in complex projective spaces, Tohoku Math. J. 55 (2003), 583-610.

[2] A. Amarzaya and Y. Ohnita, Hamiltonian stability of certain symmetric R-spaces embedded in complex Euclidean spaces, preprint, Tokyo Metropolitan University, 2002.

[3] A. Amarzaya and Y. Ohnita, Hamiltonian stability of parallel Lagrangian submanifolds embedded in complex space forms, in preparation.

[4] T. Asoh, Compact transformation groups on $\mathbf{Z}_{2}$-cohomology spheres with orbits of codimension 1, Hiroshima Math. J. 11 (1981), 571-616.

Supplement to "Compact transformation groups on $\mathbf{Z}_{2}$-cohomology spheres with orbits of codimension 1", Hiroshima Math. J. 13 (1983), 647-652.

[5] L. Bedulli and A. Gori, Homogeneous Lagrangian submanifolds, math.DG/0604169, to appear.

[6] L. Bedulli and A. Gori, A Hamiltonian stable minimal Lagrangian submanifolds of projective spaces with non-parallel second fundamental form, math.DG/0603528, to appear.

[7] L. Biliotti, Hamiltonian actions and homogeneous Lagrangian submanifolds, math.DG/0605591.

[8] N. Bourbaki, Elements de mathematique. Fasc. XXXIV. Groupes et algebres de Lie, Actualites Scientifiques et Industrielles, No. 1337, Hermann, Paris 1968.

[9] T. Cecil, Q.-S. Chi and G. R. Jensen, Isoparametric hypersurfaces with four principal curvatures, math.DG/0402272, to appear in Ann. of Math.

[10] B. Y. Chen and K. Ogiue, On totally real submanifolds, Trans. Amer. Math. Soc. 193 (1974), 257-266.

[11] P. Dazord, Sur la geometrie des sous-fibres et des feuilletages lagrangiens, (French) [On the geometry of subbundles and Lagrange foliations] Ann. Sci. École Norm. Sup. (4) 14 (1981), no. 4, 465-480 (1982).

[12] D. Ferus, H. Karcher and H. F. Münzner, Cliffordalgebren und neue isoparametrische Hyperflächen, Math. Z. 177 (1981), 479-502.

[13] V. Guillemin and R. Sjamaar, Convexity Properties of Hamiltonian Group Actions, CRM Monograph Series, Vol. 26. American Mathematical Society, Providence, RI, 2005.

[14] M. Goto and F. D. Grosshans, Semisimple Lie Algebras, Lecture Notes in Pure and Applied Mathematics, Vol. 38. Marcel Dekker, Inc., New York-Basel, 1978.

[15] S. Helgason, Differential Geometry, Lie Groups and Symmetric Spaces, Academic Press, Inc., New York, San Diego, London, Tokyo, 1978.

[16] W.-Y. Hsiang and H. B. Lawson, Jr., Minimal submanifolds of low cohomogeneity, J. Differential Geom. 5(1971), 1-38.

[17] F. Kirwan, Convexity of properties of the moment mappings, III, Invent. Math. 77 (1984), 547-552.

[18] S. Kobayashi and K. Nomizu, Foundations of Differential Geometry I, II, 1963, 1969, Wiley-Interscience, New York.

[19] M. Kotani, The first eigenvalue of homogeneous minimal hypersurfaces in a unit sphere $S^{n+1}(1)$, Tohoku Math. J. 37(1985), 523-532.

[20] W. G. McKay and J. Patera, Tables of dimensions, indices, and branching rules for representations of simple Lie algebras, Lecture Notes in Pure and Applied Mathematics, Vol. 69. Marcel Dekker, Inc., New York-Basel, 1981.

[21] H. F. Münzner, Isoparametrische Hyperfläche in Sphären, Math. Ann. 251 (1980), 5771.

[22] H. F. Münzner, Isoparametrische Hyperfläche in Sphären, II, Math. Ann. 256 (1981), 215-232.

[23] H. Muto, The first eigenvalue of the Laplacian of an isoparametric minimal hypersurface in a unit sphere, Math. Z. 197 (1988), 531-549.

[24] H. Muto, Y. Ohnita and H. Urakawa, Homogeneous minimal hypersurfaces in the unit spheres and the first eigenvalues of thier Laplacian, Tohoku Math. J. 36(1984), 253-267. 
[25] H. Naitoh, Totally real parallel submanifolds in $P^{n}(c)$, Tokyo J. Math. 4 (1981), 279306.

[26] H. Naitoh, Parallel submanifolds of complex space forms. I, Nagoya Math. J. 90 (1983), 85-117.

[27] H. Naitoh, Parallel submanifolds of complex space forms. II, Nagoya Math. J. 91 (1983), 119-149.

[28] H. Naitoh and M. Takeuchi, Totally real submanifolds and symmetric bounded domains, Osaka Math. J. 19 (1982), 717-731.

[29] Y. G. Oh, Second variation and stabilities of minimal Lagrangian submanifolds in Kähler manifolds, Invent. math. 101 (1990), 501-519.

[30] Y. G. Oh, Tight Lagrangian submanifolds in $\mathbf{C} P^{n}$, Math. Z. 207 (1991), 409-416.

[31] Y. G. Oh, Volume minimization of Lagrangian submanifolds under Hamiltonian deformations, Math. Z. 212 (1993), 175-192.

[32] Y. G. Oh, Mean curvature vector and symplectic topology of Lagrangian submanifolds in Einstein-Kähler manifolds, Math. Z. 216 (1994), 471-482.

[33] Y. Ohnita, Stability and rigidity of special Lagrangian cones over certain minimal Legendrian orbits, Osaka J. Math. 44 no.2 (2007), 305-334.

[34] H. Ozeki and M. Takeuchi, On some types of isoparametric hypersurfaces in spheres I, Tohoku Math. J.(2) 27 (1975), 515-559.

[35] H. Ozeki and M. Takeuchi, On some types of isoparametric hypersurfaces in spheres II, Tohoku Math. J.(2) 28 (1976), 7-55.

[36] B. Palmer, Buckling eigenvalues, Gauss maps and Lagrangian submanifolds, Diff. Geom. and its Appl. 4 (1994), 391-403.

[37] B. Palmer, Hamiltonian minimality and Hamiltonian stability of Gauss maps, Diff. Geom. and its Appl. 7 (1997), 51-58.

[38] M. Sato and T. Kimura, A classification of irreducible prehomogeneous vector spaces and their relative invariants, Nagoya Math. J. 65 (1977), 1-155.

[39] R. Takagi and T. Takahashi, On the principal curvatures of homogeneous hypersurfaces in a unit sphere, Differential Geometry, in honor of K. Yano, Kinokuniya, Tokyo, 1972, 469-481.

[40] M. Takeuchi, Stability of certain minimal submanifolds of compact Hermitian symmetric spaces, Tohoku Math. J. (2) 36 (1984), 293-314.

[41] S. Yamaguchi, Spectra of flag manifolds, Mem. Fac. Sci. Kyushu Univ. 33 (1979), 95112.

Department of Mathematical Sciences, Tsinghua University, Beijing 100084, P.R. CHINA

E-mail address: hma@math.tsinghua.edu.cn

Department of Mathematics, Osaka City University, Sugimoto, SumiyoshiKU, OSAKA, 558-8585, JAPAN

E-mail address: ohnita@sci.osaka-cu.ac.jp 